\title{
The Maximizing Deviation Method Based on Interval-Valued Pythagorean Fuzzy Weighted Aggregating Operator for Multiple Criteria Group Decision Analysis
}

\author{
Wei Liang, ${ }^{1,2}$ Xiaolu Zhang, ${ }^{2}$ and Manfeng Liu ${ }^{2}$ \\ ${ }^{1}$ College of Information Technology, Jiangxi University of Finance and Economics, Nanchang 330013, China \\ ${ }^{2}$ The Collaborative Innovation Center, Jiangxi University of Finance and Economics, Nanchang 330013, China \\ Correspondence should be addressed to Xiaolu Zhang; xiaolu_jy@163.com
}

Received 4 July 2015; Accepted 31 August 2015

Academic Editor: Garyfalos Papashinopoulos

Copyright (C) 2015 Wei Liang et al. This is an open access article distributed under the Creative Commons Attribution License, which permits unrestricted use, distribution, and reproduction in any medium, provided the original work is properly cited.

As a new extension of Pythagorean fuzzy set (also called Atanassov's intuitionistic fuzzy set of second type), interval-valued Pythagorean fuzzy set which is parallel to Atanassov's interval-valued intuitionistic fuzzy set has recently been developed to model imprecise and ambiguous information in practical group decision making problems. The aim of this paper is to put forward a novel decision making method for handling multiple criteria group decision making problems within interval-valued Pythagorean fuzzy environment based on interval-valued Pythagorean fuzzy numbers (IVPFNs). There are three key issues being addressed in this approach. The first is to introduce an interval-valued Pythagorean fuzzy weighted arithmetic averaging (IVPF-WAA) operator to aggregate the decision data in order to get the overall preference values of alternatives. Some desirable properties of the IVPF-WAA operator are also investigated. Based on the idea of the maximizing deviation method, the second is to establish an optimization model for determining the weights of criteria for each expert. The third is to construct a minimizing consistency optimal model to derive the weights of criteria for the group. Finally, an illustrating example is given to verify the proposed approach.

\section{Introduction}

Fuzzy set originally introduced by Zadeh [1] in 1965 is a useful tool to capture the imprecision and uncertainty in decision making $[2,3]$. It is characterized by a membership degree between zero and one, and the nonmembership degree is equal to one minus the membership degree. In 1986, Atanassov [4] extended fuzzy set to introduce the notion of Atanassov's intuitionistic fuzzy set. In Atanassov's intuitionistic fuzzy theory, the membership degree and the nonmembership degree are more or less independent; the only constraint is that the sum of the two degrees must not exceed one [5]. Atanassov's intuitionistic fuzzy sets have been broadly applied in real-life multiple criteria decision making (MCDM) $[6,7]$ or multiple criteria group decision making (MCGDM) problems [8]. For example, Wang et al. [6] developed a method based on Atanassov's intuitionistic fuzzy dependent aggregation operators for solving the supplier selection problem. Wang and Zhang [7] proposed an evidential reasoning-based decision making method for handling Atanassov's intuitionistic fuzzy MCDM problems with incomplete weight information, and so forth. Deschrijver and Kerre [5] investigated the position of Atanassov's intuitionistic fuzzy set theory in the framework of the different theories modelling imprecision.

In the beginning of the 1990s, Atanassov [9] further proposed the concept of Atanassov's intuitionistic fuzzy set of second type as a useful extension of Atanassov's intuitionistic fuzzy set. Yager [10] called it Pythagorean fuzzy set (PFS). For simplicity, this paper still employs the term "PFS" to denote Atanassov's intuitionistic fuzzy set of second type in the decision process. The main difference between PFS and Atanassov's intuitionistic fuzzy set is that the former is required to satisfy the situation that the square sum of the membership degree and the nonmembership degree is equal to or less than one, but the sum of the two degrees 
is not required to be equal to or less than one, while the latter is required to satisfy the situation that the sum of the two degrees is equal to or less than one. Ever since PFSs' appearance, many researchers have paid great attention to the decision making problems with Pythagorean fuzzy information. For instance, Yager [10] developed a useful decision approach based on Pythagorean fuzzy aggregation operations to handle the MCDM problems with Pythagorean fuzzy information. Zhang and Xu [11] provided the detailed mathematical expressions for PFSs and presented the concept of Pythagorean fuzzy number (PFN). Meanwhile, they also proposed a Pythagorean fuzzy TOPSIS (Technique for Order Preference by Similarity to Ideal Solution) for handling the MCDM problem within PFNs. Yager and Abbasov [12] showed that the PFNs are a subclass of complex numbers called $\pi-i$ numbers and proposed a decision method to handle the MCDM problem in which the criterion values are expressed by $\pi-i$ numbers. Afterwards, Beliakov and James [13] focused on how the notion of "averaging" should be treated in the case of PFNs. Reformat and Yager [14] applied the PFSs in handling the collaborative-based recommender system. In addition, Atanassov et al. [15] extended the concept of Atanassov's intuitionistic fuzzy set of second type to present the concept of Atanassov's intuitionistic fuzzy set of p-type.

In human cognitive and decision making activities, it is not completely justifiable or technically sound to quantify the degrees of the membership and nonmembership in terms of a single numeric value $[16,17]$. To this end, Zhang [18] further extended the PFSs to propose the concept of interval-valued PFSs (IVPFSs) which is parallel to Atanassov's intervalvalued intuitionistic fuzzy set $[19,20]$. The IVPFS can also be called Atanassov's interval-valued intuitionistic fuzzy set of second type as a particular answer to the open problem proposed by Atanassov $[9,21]$ of how to define a combination between Atanassov's intuitionistic fuzzy set of second type and Atanassov's interval-valued intuitionistic fuzzy set. The elements in IVPFS are called interval-valued Pythagorean fuzzy numbers (IVPFNs). Considering the fact that IVPFNs have great powerful ability to model the imprecise and ambiguous information in real-world applications [18], this paper develops a maximizing deviation method based on interval-valued Pythagorean fuzzy weighted average aggregating (IVPF-WAA) operator to solve MCGDM problems with IVPFNs. We first present the concept of the score and accuracy functions for IVPFNs, and we further present a score and accuracy functions-based ranking method for comparing the magnitude of IVPFNs. Next, we employ the maximizing deviation method to determine the weights of criteria for each expert. Meanwhile, we also construct a minimizing consistency optimal model to derive the weights of criteria for the group. Afterwards, we define an IVPFWAA operator and investigate its useful properties. Using IVPF-WAA operator, all individual decision matrices are aggregated into the collective decision matrix, and further the comprehensive values of alternatives are obtained. Using the proposed ranking method of IVPFNs, the ranking orders of all alternatives are obtained. At length, we provide a risk evaluation case of technological innovation in high-tech enterprises to validate the effectiveness and applicability of the proposed decision method.

This paper is organized as follows. Section 2 briefly reviews some concepts of PFSs as well as IVPFSs and also presents a new ranking method for IVPFNs. Section 3 develops a new group decision method to handle the MCGDM problems with IVPFNs. Section 4 provides a practical decision problem to demonstrate the implementation process of the proposed method. Section 5 presents our conclusions.

\section{Preliminaries}

The basic concepts of PFNs and IVPFNs are briefly reviewed in this section. Afterwards, novel score and accuracy functions for IVPFNs are proposed. Furthermore, a new comparison method for IVPFNs is developed.

Definition 1 (see $[9,11])$. Let $X$ be a fix set. A PFS $P$ is an object having the form

$$
P=\left\{\left\langle x, P\left(\mu_{P}(x), v_{P}(x)\right)\right\rangle \mid x \in X\right\},
$$

where the function $\mu_{P}: X \rightarrow[0,1]$ defines the degree of membership and $v_{P}: X \rightarrow[0,1]$ defines the degree of nonmembership of the element $x \in X$ to $P$, respectively, and, for every $x \in X$, it holds that

$$
\left(\mu_{P}(x)\right)^{2}+\left(v_{P}(x)\right)^{2} \leq 1
$$

Definition 2 (see $[9,11])$. Let $\beta_{1}=P\left(\mu_{\beta_{1}}, v_{\beta_{1}}\right), \beta_{2}=$ $P\left(\mu_{\beta_{2}}, v_{\beta_{2}}\right)$, and $\beta=P\left(\mu_{\beta}, v_{\beta}\right)$ be three PFNs, and five basic operations on them are defined as follows:

(1) $\beta_{1} \oplus \beta_{2}=P\left(\sqrt{\mu_{\beta_{1}}^{2}+\mu_{\beta_{2}}^{2}-\mu_{\beta_{1}}^{2} \mu_{\beta_{2}}^{2}}, v_{\beta_{1}} v_{\beta_{2}}\right)$;

(2) $\beta_{1} \otimes \beta_{2}=P\left(\mu_{\beta_{1}} \mu_{\beta_{2}}, \sqrt{v_{\beta_{1}}^{2}+v_{\beta_{2}}^{2}-v_{\beta_{1}}^{2} v_{\beta_{2}}^{2}}\right)$;

(3) $\lambda \beta=P\left(\sqrt{1-\left(1-\mu_{\beta}^{2}\right)^{\lambda}},\left(v_{\beta}\right)^{\lambda}\right),(\lambda>0)$;

(4) $\beta^{\lambda}=P\left(\left(\mu_{\beta}\right)^{\lambda}, \sqrt{1-\left(1-v_{\beta}^{2}\right)^{\lambda}}\right), \quad(\lambda>0)$;

(5) $\beta^{c}=P\left(v_{\beta}, \mu_{\beta}\right)$.

In many real-world decision problems, the values of the membership function and nonmembership function in a PFS are difficult for the decision maker to assign exact numbers. Zhang [18] suggested that the decision maker can employ intervals to express his/her preference about the membership function and the nonmembership function in a PFS and extended the concept of PFS to propose the concept of IVPFS. Its definition is introduced as follows.

Definition 3 (see [18]). Let $X$ be a fix set, and an IVPFS $\widetilde{P}$ over $X$ is an object having the following mathematic form:

$$
\widetilde{P}=\left\{\left\langle x, \widetilde{P}\left(\widetilde{\mu}_{\widetilde{P}}(x), \widetilde{v}_{\widetilde{P}}(x)\right)\right\rangle \mid x \in X\right\},
$$

where $\tilde{\mu}_{\widetilde{P}}(x)\left(=\left[\widetilde{\mu}_{\widetilde{P}}^{L}(x), \widetilde{\mu}_{\widetilde{P}}^{U}(x)\right]\right) \subseteq[0,1]$ and $\widetilde{v}_{\widetilde{P}}(x)(=$ $\left.\left[\widetilde{v}_{\widetilde{P}}^{L}(x), \widetilde{v}_{\widetilde{P}}^{U}(x)\right]\right) \subseteq[0,1]$ are interval values and $\tilde{\mu}_{\widetilde{P}}^{L}(x), \widetilde{\mu}_{\widetilde{P}}^{U}(x)$, $\widetilde{v}_{\widetilde{P}}^{L}(x), \widetilde{v}_{\widetilde{P}}^{U}(x) \in[0,1]$ and $\left(\widetilde{\mu}_{\widetilde{P}}^{U}(x)\right)^{2}+\left(\widetilde{v}_{\widetilde{P}}^{U}(x)\right)^{2} \leq 1$. 
Clearly, the IVPFS reduces to a PFS if $\tilde{\mu}_{\widetilde{P}}^{L}(x)=\widetilde{\mu}_{\widetilde{P}}^{U}(x)$ and $\widetilde{v}_{\tilde{P}}^{L}(x)=\widetilde{v}_{\widetilde{P}}^{U}(x)$, and the IVPFS reduces to Atanassov's interval-valued intuitionistic fuzzy set if $\widetilde{\mu}_{\widetilde{P}}^{U}(x)+\widetilde{v}_{\widetilde{P}}^{U}(x) \leq 1$. For simplicity, $\widetilde{P}\left(\widetilde{\mu}_{\widetilde{P}}(x), \widetilde{v}_{\widetilde{P}}(x)\right)$ is called an IVPFN denoted by $\widetilde{\beta}=\widetilde{P}\left(\widetilde{\mu}_{\tilde{\beta}}, \widetilde{v}_{\tilde{\beta}}\right)$, where $\mu_{\tilde{\beta}}\left(=\left[\widetilde{\mu}_{\widetilde{\beta}}^{L}, \widetilde{\mu}_{\widetilde{\beta}}^{U}\right]\right) \subset[0,1], \widetilde{v}_{\tilde{\beta}}\left(=\left[\widetilde{v}_{\widetilde{\beta}}^{L}, \widetilde{v}_{\widetilde{\beta}}^{U}\right]\right) \subset$ $[0,1]$, and $\left(\widetilde{\mu}_{\tilde{\beta}}^{U}\right)^{2}+\left(\widetilde{v}_{\tilde{\beta}}^{U}\right)^{2} \leq 1$. It is noted that the IVPFN $\widetilde{\beta}=\widetilde{P}\left(\left[\tilde{\mu}_{\tilde{\beta}}^{L}, \widetilde{\mu}_{\widetilde{\beta}}^{U}\right],\left[\widetilde{v}_{\widetilde{\beta}}^{L}, \widetilde{v}_{\widetilde{\beta}}^{U}\right]\right)$ is called Atanassov's interval-valued intuitionistic fuzzy number if $\widetilde{\mu}_{\tilde{\beta}}^{U}+\widetilde{v}_{\tilde{\beta}}^{U} \leq 1$.

Example 4. Let $X=\left\{x_{1}, x_{2}, x_{3}\right\}$, and let $\widetilde{P}\left(x_{1}\right)=$ $\widetilde{P}([0.8,0.9],[0.3,0.4]), \widetilde{P}\left(x_{2}\right)=\widetilde{P}([0.5,0.7],[0.4,0.6])$, and $\widetilde{P}\left(x_{3}\right)=\widetilde{P}([0.7,0.8],[0.2,0.4])$ be three IVPFNs of $x_{i}(i=$ $1,2,3)$ to a set $\widetilde{P}$. Thus, $\widetilde{P}$ can be called an IVPFS which is denoted as follows:

$$
\begin{gathered}
\widetilde{P}=\left\{\left\langle x_{1}, \widetilde{P}([0.8,0.9],[0.3,0.4])\right\rangle,\right. \\
\left\langle x_{2}, \widetilde{P}([0.5,0.7],[0.4,0.6])\right\rangle, \\
\left.\left\langle x_{3}, \widetilde{P}([0.7,0.8],[0.2,0.4])\right\rangle\right\} .
\end{gathered}
$$

Remark 5. It is noted that the main difference between IVPFN and Atanassov's interval-valued intuitionistic fuzzy number is their different constraint conditions. The space of the constraint condition of IVPFN is usually greater than the space of the constraint condition of Atanassov's interval-valued intuitionistic fuzzy number. In other words, the IVPFN can not only model the uncertain situations which Atanassov's interval-valued intuitionistic fuzzy number can capture where the sum of $\widetilde{\mu}_{\tilde{\beta}}^{U}$ and $\widetilde{v}_{\tilde{\beta}}^{U}$ is equal to or less than 1, but also model some other situations which Atanassov's interval-valued intuitionistic fuzzy number cannot describe where the sum of $\widetilde{\mu}_{\tilde{\beta}}^{U}$ and $\widetilde{v}_{\widetilde{\beta}}^{U}$ is bigger than 1 but their square sum is equal to or less than 1 .

Definition 6 (see [18]). Let $\widetilde{\beta}=\widetilde{P}\left(\left[\widetilde{\mu}_{\tilde{\beta}}^{L}, \widetilde{\mu}_{\tilde{\beta}}^{U}\right],\left[\widetilde{v}_{\widetilde{\beta}}^{L}, \widetilde{v}_{\tilde{\beta}}^{U}\right]\right), \widetilde{\beta}_{1}=$ $\widetilde{P}\left(\left[\tilde{\mu}_{\tilde{\beta}_{1}}^{L}, \widetilde{\mu}_{\widetilde{\beta}_{1}}^{U}\right],\left[\widetilde{v}_{\tilde{\beta}_{1}}^{L}, \widetilde{v}_{\widetilde{\beta}_{1}}^{U}\right]\right)$, and $\widetilde{\beta}_{2}=\widetilde{P}\left(\left[\tilde{\mu}_{\tilde{\beta}_{2}}^{L}, \widetilde{\mu}_{\widetilde{\beta}_{2}}^{U}\right],\left[\widetilde{v}_{\tilde{\beta}_{2}}^{L}, \widetilde{v}_{\widetilde{\beta}_{2}}^{U}\right]\right)$ be three IVPFNs. For $\lambda(\lambda>0)$ represents a scalar mathematical operator, the basic operations on them are defined as follows:

(1) $\widetilde{\beta}_{1} \oplus \widetilde{\beta}_{2}$

$$
\begin{aligned}
& =\widetilde{P}\left(\left[\sqrt{\left(\tilde{\mu}_{\tilde{\beta}_{1}}^{L}\right)^{2}+\left(\tilde{\mu}_{\tilde{\beta}_{2}}^{L}\right)^{2}-\left(\tilde{\mu}_{\tilde{\beta}_{1}}^{L}\right)^{2}\left(\tilde{\mu}_{\tilde{\beta}_{2}}^{L}\right)^{2}},\right.\right. \\
& \left.\sqrt{\left(\widetilde{\mu}_{\tilde{\beta}_{1}}^{U}\right)^{2}+\left(\widetilde{\mu}_{\tilde{\beta}_{2}}^{U}\right)^{2}-\left(\widetilde{\mu}_{\tilde{\beta}_{1}}^{U}\right)^{2}\left(\widetilde{\mu}_{\tilde{\beta}_{2}}^{U}\right)^{2}}\right],\left[\widetilde{v}_{\widetilde{\beta}_{1}}^{L} \widetilde{v}_{\widetilde{\beta}_{2}}^{L},\right. \\
& \left.\left.\widetilde{v}_{\widetilde{\beta}_{1}}^{U} \widetilde{v}_{\widetilde{\beta}_{2}}^{U}\right]\right) ;
\end{aligned}
$$

(2) $\widetilde{\beta}_{1} \otimes \widetilde{\beta}_{2}=\widetilde{P}\left(\left[\tilde{\mu}_{\tilde{\beta}_{1}}^{L} \widetilde{\mu}_{\widetilde{\beta}_{2}}^{L}, \widetilde{\mu}_{\widetilde{\beta}_{1}}^{U} \widetilde{\beta}_{\widetilde{\beta}_{2}}^{U}\right]\right.$,

$\left[\sqrt{\left(\widetilde{v}_{\tilde{\beta}_{1}}^{L}\right)^{2}+\left(\widetilde{v}_{\tilde{\beta}_{2}}^{L}\right)^{2}-\left(\widetilde{v}_{\tilde{\beta}_{1}}^{L}\right)^{2}\left(\widetilde{v}_{\tilde{\beta}_{2}}^{L}\right)^{2}}\right.$,

$\left.\left.\sqrt{\left(\widetilde{v}_{\widetilde{\beta}_{1}}^{U}\right)^{2}+\left(\widetilde{v}_{\widetilde{\beta}_{2}}^{U}\right)^{2}-\left(\widetilde{v}_{\tilde{\beta}_{1}}^{U}\right)^{2}\left(\widetilde{v}_{\widetilde{\beta}_{2}}^{U}\right)^{2}}\right]\right) ;$

(3) $\lambda \widetilde{\beta}=\widetilde{P}\left(\left[\sqrt{1-\left(1-\left(\widetilde{\mu}_{\tilde{\beta}}^{L}\right)^{2}\right)^{\lambda}}\right.\right.$,

$\left.\left.\sqrt{1-\left(1-\left(\widetilde{\mu}_{\tilde{\beta}}^{U}\right)^{2}\right)^{\lambda}}\right],\left[\left(\widetilde{v}_{\tilde{\beta}}^{L}\right)^{\lambda},\left(\widetilde{v}_{\widetilde{\beta}}^{U}\right)^{\lambda}\right]\right) ;$

(4) $(\widetilde{\beta})^{\lambda}=\widetilde{P}\left(\left[\left(\tilde{\mu}_{\tilde{\beta}}^{L}\right)^{\lambda},\left(\tilde{\mu}_{\tilde{\beta}}^{U}\right)^{\lambda}\right]\right.$,

$\left.\left[\sqrt{1-\left(1-\left(\widetilde{v}_{\tilde{\beta}}^{L}\right)^{2}\right)^{\lambda}}, \sqrt{1-\left(1-\left(\widetilde{v}_{\tilde{\beta}}^{U}\right)^{2}\right)^{\lambda}}\right]\right) ;$

(5) $(\widetilde{\beta})^{c}=\widetilde{P}\left(\left[\widetilde{v}_{\widetilde{\beta}}^{L}, \widetilde{v}_{\widetilde{\beta}}^{U}\right],\left[\widetilde{\mu}_{\tilde{\beta}}^{L}, \widetilde{\mu}_{\widetilde{\beta}}^{U}\right]\right)$.

Definition 7 (see [18]). Let $\widetilde{\beta}_{j}=\widetilde{P}\left(\left[\tilde{\mu}_{\widetilde{\beta}_{j}}^{L}, \widetilde{\mu}_{\tilde{\beta}_{j}}^{U}\right]\right.$, $\left.\left[\widetilde{v}_{\widetilde{\beta}_{j}}^{L}, \widetilde{v}_{\widetilde{\beta}_{j}}^{U}\right]\right)(j=$ $1,2)$ be two IVPFNs, and a nature quasi-ordering on the IVPFNs is defined as follows:

$$
\begin{aligned}
& \widetilde{\beta}_{1} \geq \widetilde{\beta}_{2} \\
& \text { iff } \widetilde{\mu}_{\widetilde{\beta}_{1}}^{L} \geq \widetilde{\mu}_{\widetilde{\beta}_{2}}^{L}, \\
& \widetilde{\mu}_{\widetilde{\beta}_{1}}^{U} \geq \widetilde{\mu}_{\widetilde{\beta}_{\beta_{2}}}^{U}, \\
& \widetilde{v}_{\widetilde{\beta}_{1}}^{L} \leq \widetilde{v}_{\widetilde{\beta}_{2}}^{L}, \\
& \widetilde{v}_{\widetilde{\beta}_{1}}^{U} \leq \widetilde{v}_{\widetilde{\beta}_{2}}^{U} .
\end{aligned}
$$

In the following, we present a score function and an accuracy function for IVPFNs.

Definition 8. Let $\widetilde{\beta}=\widetilde{P}\left(\left[\widetilde{\mu}_{\tilde{\beta}}^{L}, \widetilde{\mu}_{\tilde{\beta}}^{U}\right],\left[\widetilde{v}_{\tilde{\beta}}^{L}, \widetilde{v}_{\tilde{\beta}}^{U}\right]\right)$ be an IVPFN; the score function of $\widetilde{\beta}$ is defined as

$$
s(\widetilde{\beta})=\frac{1}{2}\left(\left(\tilde{\mu}_{\tilde{\beta}}^{L}\right)^{2}-\left(\widetilde{v}_{\tilde{\beta}}^{L}\right)^{2}+\left(\widetilde{\mu}_{\tilde{\beta}}^{U}\right)^{2}-\left(\widetilde{v}_{\tilde{\beta}}^{U}\right)^{2}\right)
$$

and the accuracy function of $\widetilde{\beta}$ is defined as follows:

$$
h(\widetilde{\beta})=\frac{1}{2}\left(\left(\widetilde{\mu}_{\tilde{\beta}}^{L}\right)^{2}+\left(\widetilde{v}_{\tilde{\beta}}^{L}\right)^{2}+\left(\widetilde{\mu}_{\tilde{\beta}}^{U}\right)^{2}+\left(\widetilde{v}_{\widetilde{\beta}}^{U}\right)^{2}\right) .
$$

Based on the concepts of the score and accuracy functions of IVPFNs, we introduce a ranking method for comparing the magnitude of IVPFNs. 
Definition 9. Let $\widetilde{\beta}_{j}=\widetilde{P}\left(\left[\tilde{\mu}_{\tilde{\beta}_{j}}^{L}, \widetilde{\mu}_{\tilde{\beta}_{j}}^{U}\right]\right.$, $\left.\left[\widetilde{v}_{\tilde{\beta}_{j}}^{L}, \widetilde{v}_{\tilde{\beta}_{j}}^{U}\right]\right)(j=1,2)$ be two IVPFNs, let $s\left(\widetilde{\beta}_{1}\right)$ and $s\left(\widetilde{\beta}_{2}\right)$ be the score values of $\widetilde{\beta}_{1}$ and $\widetilde{\beta}_{2}$, respectively, and let $h\left(\widetilde{\beta}_{1}\right)$ and $h\left(\widetilde{\beta}_{2}\right)$ be the accuracy values of $\widetilde{\beta}_{1}$ and $\widetilde{\beta}_{2}$, respectively, and then

(1) if $s\left(\widetilde{\beta}_{1}\right)<s\left(\widetilde{\beta}_{2}\right)$, then $\widetilde{\beta}_{1}<\widetilde{\beta}_{2}$;

(2) if $s\left(\widetilde{\beta}_{1}\right)=s\left(\widetilde{\beta}_{2}\right)$, then $\left\{h\left(\widetilde{\beta}_{1}\right)<h\left(\widetilde{\beta}_{2}\right) \Rightarrow \widetilde{\beta}_{1}<\right.$ $\widetilde{\beta}_{2}, h\left(\widetilde{\beta}_{1}\right)=h\left(\widetilde{\beta}_{2}\right) \Rightarrow \widetilde{\beta}_{1} \sim \widetilde{\beta}_{2}, h\left(\widetilde{\beta}_{1}\right)>h\left(\widetilde{\beta}_{2}\right) \Rightarrow$ $\left.\widetilde{\beta}_{1}>\widetilde{\beta}_{2}\right\} ;$

(3) if $s\left(\widetilde{\beta}_{1}\right)>s\left(\widetilde{\beta}_{2}\right)$, then $\widetilde{\beta}_{1}>\widetilde{\beta}_{2}$.

Example 10. For two IVPFNs, $\widetilde{\beta}_{1}=\widetilde{P}([\sqrt{7} / 4, \sqrt{11} / 4],[1 / 4$, $\sqrt{5} / 4])$ and $\widetilde{\beta}_{2}=\widetilde{P}([\sqrt{9} / 4, \sqrt{10} / 4],[\sqrt{2} / 4, \sqrt{5} / 4])$, the following results based on Definition 8 are obtained:

$$
\begin{aligned}
& s\left(\widetilde{\beta}_{1}\right)=\frac{1}{2}\left(\frac{7}{16}-\frac{1}{16}+\frac{11}{16}-\frac{5}{16}\right)=\frac{3}{8}, \\
& s\left(\widetilde{\beta}_{2}\right)=\frac{1}{2}\left(\frac{9}{16}-\frac{2}{16}+\frac{10}{16}-\frac{5}{16}\right)=\frac{3}{8}, \\
& h\left(\widetilde{\beta}_{1}\right)=\frac{1}{2}\left(\frac{7}{16}+\frac{1}{16}+\frac{11}{16}+\frac{5}{16}\right)=\frac{3}{4}, \\
& h\left(\widetilde{\beta}_{2}\right)=\frac{1}{2}\left(\frac{9}{16}+\frac{2}{16}+\frac{10}{16}+\frac{5}{16}\right)=\frac{13}{16} .
\end{aligned}
$$

According to Definition 9, it is easy to obtain $\widetilde{\beta}_{1} \prec \widetilde{\beta}_{2}$.

The interval-valued Pythagorean fuzzy distance measure is introduced as follows.

Definition 11 (see [18]). Let $\widetilde{\beta}_{j}=\widetilde{P}\left(\left[\widetilde{\mu}_{\widetilde{\beta}_{j}}^{L}, \widetilde{\mu}_{\widetilde{\beta}_{j}}^{U}\right],\left[\widetilde{v}_{\widetilde{\beta}_{j}}^{L}, \widetilde{v}_{\widetilde{\beta}_{j}}^{U}\right]\right)(j=$ $1,2)$ be two IVPFNs, and then the distance between $\widetilde{\beta}_{1}$ and $\widetilde{\beta}_{2}$ is defined as follows:

$$
\begin{aligned}
& d\left(\widetilde{\beta}_{1}, \widetilde{\beta}_{2}\right)=\frac{1}{4}\left(\left|\left(\widetilde{\mu}_{\tilde{\beta}_{1}}^{L}\right)^{2}-\left(\tilde{\mu}_{\tilde{\beta}_{2}}^{L}\right)^{2}\right|\right. \\
& +\left|\left(\widetilde{\mu}_{\widetilde{\beta}_{1}}\right)^{2}-\left(\widetilde{\mu}_{\widetilde{\beta}_{2}}\right)^{2}\right|+\left|\left(\widetilde{v}_{\widetilde{\beta}_{1}}^{L}\right)^{2}-\left(\widetilde{v}_{\widetilde{\beta}_{2}}^{L}\right)^{2}\right| \\
& +\left|\left(\widetilde{v}_{\widetilde{\beta}_{1}}^{U}\right)^{2}-\left(\widetilde{v}_{\widetilde{\beta}_{2}}^{U}\right)^{2}\right|+\left|\left(\tilde{\pi}_{\widetilde{\beta}_{1}}^{L}\right)^{2}-\left(\tilde{\pi}_{\widetilde{\beta}_{2}}^{L}\right)^{2}\right| \\
& \left.+\left|\left(\tilde{\pi}_{\tilde{\beta}_{1}}^{U}\right)^{2}-\left(\tilde{\pi}_{\tilde{\beta}_{2}}^{U}\right)^{2}\right|\right) \text {. }
\end{aligned}
$$

Example 12. For two IVPFNs, $\widetilde{\beta}_{1}=\widetilde{P}([0.6,0.8],[0.3,0.5])$ and $\widetilde{\beta}_{2}=\widetilde{P}([0.8,0.9],[0.2,0.3])$, the following result based on Definition 11 is obtained:

$$
\begin{aligned}
d & \left(\widetilde{\beta}_{1}, \widetilde{\beta}_{2}\right)=\frac{1}{4}\left(\left|0.6^{2}-0.8^{2}\right|+\left|0.8^{2}-0.9^{2}\right|\right. \\
& +\left|0.3^{2}-0.2^{2}\right|+\left|0.5^{2}-0.3^{2}\right| \\
& +\left|1-0.8^{2}-0.5^{2}-\left(1-0.9^{2}-0.3^{2}\right)\right| \\
& \left.+\left|1-0.6^{2}-0.3^{2}-\left(1-0.8^{2}-0.2^{2}\right)\right|\right)=0.225 .
\end{aligned}
$$

\section{The Maximizing Deviation Method Based on IVPF-WAA Operator}

This section first introduces an MCGDM problem under interval-valued Pythagorean fuzzy environment. Then, the maximizing deviation model is established to determine the weights of criteria. Afterwards, the IVPF-WAA operator is presented to aggregate the given decision information and the decision can be made. Finally, an algorithm of the proposed method is introduced.

3.1. Problem Formulation. Consider an MCGDM problem under interval-valued Pythagorean fuzzy environment; let $\mathbf{A}=\left\{A_{1}, A_{2}, \ldots, A_{m}\right\}$ be a discrete set of $m(m \geq 2)$ feasible alternatives and let $\mathbf{C}=\left\{C_{1}, C_{2}, \ldots, C_{n}\right\}$ be a finite set of criteria. Let $E=\left\{e_{1}, e_{2}, \ldots, e_{g}\right\}$ be a group of experts, and let $\lambda=\left(\lambda_{1}, \lambda_{2}, \ldots, \lambda_{g}\right)^{T}$ be the weight vector of experts, where $0 \leq \lambda_{k} \leq 1$ and $\sum_{k=1}^{g} \lambda_{k}=1$. We denote the weight vector of criteria for the expert $e_{k}$ by $\mathbf{w}^{k}=\left(w_{1}^{k}, w_{2}^{k}, \ldots, w_{n}^{k}\right)^{T}$. Without loss of generality, in this paper we suppose that the criteria weights are completely unknown or partially known beforehand, and the experts' weights are completely known in advance. The expert $e_{k}$ employs the IVPFN $\widetilde{\beta}_{i j}^{k}$ to express the criterion value of the alternative $A_{i}$ with respect to the criterion $C_{j}$.

Definition 13. The matrix $\mathscr{P}^{k}=\left(\widetilde{\beta}_{i j}^{k}\right)_{m \times n}$ is called an intervalvalued Pythagorean fuzzy decision matrix if all entries of the matrix $\mathscr{P}^{k}$ are IVPFNs; that is, $\widetilde{\beta}_{i j}^{k}=\widetilde{P}\left(\left[\widetilde{u}_{\tilde{\beta}_{i j}^{k}}^{L}, \widetilde{u}_{\widetilde{\beta}_{i j}^{k}}^{U}\right],\left[\widetilde{v}_{\tilde{\beta}_{i j}^{k}}^{L}, \widetilde{v}_{\widetilde{i}_{i j}^{k}}^{U}\right]\right)$.

Therefore, the MCGDM problem with IVPFNs can be concisely expressed in interval-valued Pythagorean fuzzy decision matrix as follows:

$$
\begin{aligned}
\mathscr{P}^{k}=\left(\widetilde{\beta}_{i j}^{k}\right)_{m \times n}=\left(\widetilde{P}\left(\left[\widetilde{u}_{\tilde{\beta}_{i j}^{k}}^{L} \widetilde{u}_{\widetilde{\beta}_{i j}^{k}}^{U}\right],\left[\widetilde{v}_{\tilde{\beta}_{i j}^{k}}^{L} \widetilde{v}_{\widetilde{\beta}_{i j}^{k}}^{U}\right]\right)\right)_{m \times n}, \\
\\
(k \in\{1,2, \ldots, g\}) .
\end{aligned}
$$

The element $\widetilde{\beta}_{i j}^{k}$ in the matrix $\mathscr{P}^{k}$ indicates that the alternative $A_{i}$ is an excellent alternative for the expert $e_{k}$ on the criterion $C_{j}$ with a margin $\left[\tilde{u}_{\tilde{\beta}_{i j}^{k}}^{L}, \widetilde{u}_{\tilde{\beta}_{i j}^{k}}^{U}\right]$ and simultaneously the alternative $A_{i}$ is not an excellent choice with a chance $\left[\widetilde{v}_{\widetilde{\beta}_{i j}^{k}}^{L}, \widetilde{v}_{\tilde{\beta}_{i j}^{k}}^{U}\right]$.

3.2. The Maximizing Deviation Model for Determining the Optimal Weights. The maximizing deviation method originally proposed by Wang [22] is used to determine the weights of criteria for solving MCDM problems with crisp (nonfuzzy) numbers. This paper employs the main structure of the maximizing deviation method to establish an optimization model for determining the optimal weights of criteria under interval-valued Pythagorean fuzzy environment. At the beginning, we employ the interval-valued Pythagorean fuzzy distance measure (i.e., (10)) to calculate the deviations between each alternative and other alternatives. 
Definition 14. For the criterion $C_{j}(j \in\{1,2, \ldots, n\})$ and the expert $e_{k}(k \in\{1,2, \ldots, g\})$, the deviation value between the alternative $A_{\xi}$ and the alternative $A_{\zeta}(\xi \neq \zeta)$ is defined as follows:

$$
\begin{aligned}
& D_{\xi \zeta j}^{k}=w_{j}^{k} d\left(\widetilde{\beta}_{\xi j}^{k}, \widetilde{\beta}_{\zeta j}^{k}\right)=\frac{1}{4} w_{j}^{k}\left(\left|\left(\widetilde{\mu}_{\widetilde{\beta}_{\xi j}^{k}}^{L}\right)^{2}-\left(\widetilde{\mu}_{\widetilde{\beta}_{\zeta j}^{k}}^{L}\right)^{2}\right|\right. \\
& +\left|\left(\widetilde{\mu}_{\widetilde{\beta}_{\xi j}^{k}}^{U}\right)^{2}-\left(\widetilde{\mu}_{\widetilde{\beta}_{\zeta j}^{k}}^{U}\right)^{2}\right|+\left|\left(\widetilde{v}_{\widetilde{\beta}_{\xi j}^{k}}^{L}\right)^{2}-\left(\widetilde{v}_{\widetilde{\beta}_{\zeta j}^{k}}^{L}\right)^{2}\right| \\
& +\left|\left(\widetilde{v}_{\widetilde{\beta}_{\xi j}^{k}}^{U}\right)^{2}-\left(\widetilde{v}_{\widetilde{\beta}_{\zeta j}^{k}}^{U}\right)^{2}\right|+\left|\left(\widetilde{\pi}_{\widetilde{\beta}_{\xi j}^{k}}^{L}\right)^{2}-\left(\widetilde{\pi}_{\widetilde{\beta}_{\zeta j}^{k}}^{L}\right)^{2}\right| \\
& \left.+\left|\left(\widetilde{\pi}_{\widetilde{\beta}_{\xi j}^{k}}^{U}\right)^{2}-\left(\widetilde{\pi}_{\widetilde{\beta}_{\zeta j}^{k}}^{U}\right)^{2}\right|\right) .
\end{aligned}
$$

Then, for the criterion $C_{j}(j \in\{1,2, \ldots, n\})$ and the expert $e_{k}(k \in\{1,2, \ldots, g\})$, the deviation value between the alternative $A_{i}(i \in\{1,2, \ldots, m\})$ and all the other alternatives can be computed as

$$
D_{\xi j}^{k}=\sum_{\zeta=1}^{m} w_{j}^{k} d\left(\widetilde{\beta}_{\xi j}^{k}, \widetilde{\beta}_{\zeta j}^{k}\right) .
$$

Furthermore, for the criterion $C_{j}(j \in\{1,2, \ldots, n\})$ and the expert $e_{k}(k \in\{1,2, \ldots, g\})$, the deviation value of all the alternatives to the other alternatives can be calculated as follows:

$$
D_{j}^{k}=\sum_{\xi=1}^{m} \sum_{\zeta=1}^{m} D_{\xi j}^{k}=\sum_{\xi=1}^{m} \sum_{\zeta=1}^{m} w_{j}^{k} d\left(\widetilde{\beta}_{\xi j}^{k}, \widetilde{\beta}_{\zeta j}^{k}\right) .
$$

According to the literature [22-25], for an MCDM problem, if the criterion values of all alternatives have small differences under a criterion, it is easy to see that such a criterion plays a less important role in the priority procedure, while if the criterion values of all alternatives have obvious differences, then this criterion plays a more important role in choosing the best alternative. That is to say, from the standpoint of ranking the alternatives, if one criterion has similar criterion values across alternatives, it should be assigned a small weight; otherwise, the criterion which makes larger deviations should be assigned a bigger weight, in spite of the degree of its own importance. In particular, if all alternatives score equally with respect to a given criterion, then such a criterion will be judged as unimportant by most of the experts and would be assigned zero weight.
To this end, we establish an optimal model which maximizes all deviation values for all the criteria to select the weight vector $\mathbf{w}^{k}$ for the expert $e_{k}(k \in\{1,2, \ldots, g\})$ as follows:

$$
\begin{aligned}
\max & D^{k}=\sum_{j=1}^{n} \sum_{\xi=1}^{m} \sum_{\zeta=1}^{m} w_{j}^{k} d\left(\widetilde{\beta}_{\xi j}^{k}, \widetilde{\beta}_{\zeta j}^{k}\right) \\
\text { s.t. } & \sum_{j=1}^{n}\left(w_{j}^{k}\right)^{2}=1, \\
& w_{j}^{k} \geq 0, j \in\{1,2, \ldots, n\} .
\end{aligned}
$$

The Lagrange function of the optimization model (MOD-1) can be obtained as follows:

$$
\begin{aligned}
L\left(\mathbf{w}^{k}, \eta\right)= & \sum_{j=1}^{n} \sum_{\xi=1}^{m} \sum_{\zeta=1}^{m} w_{j}^{k} d\left(\widetilde{\beta}_{\xi j}^{k}, \widetilde{\beta}_{\zeta j}^{k}\right) \\
& +\frac{\eta}{2}\left(\sum_{j=1}^{n}\left(w_{j}^{k}\right)^{2}-1\right),
\end{aligned}
$$

where $\eta$ is a real number, denoting the Lagrange multiplier variable.

Then, the partial derivatives of $L$ are computed as

$$
\begin{aligned}
\frac{\partial L}{\partial w_{j}^{k}} & =\sum_{\xi=1}^{m} \sum_{\zeta=1}^{m} d\left(\widetilde{\beta}_{\xi j}^{k}, \widetilde{\beta}_{\zeta j}^{k}\right)+\eta w_{j}^{k}=0, \\
\frac{\partial L}{\partial \eta} & =\frac{1}{2}\left(\sum_{j=1}^{n}\left(w_{j}^{k}\right)^{2}-1\right)=0 .
\end{aligned}
$$

By (17), we can get

$$
w_{j}^{k}=\frac{\sum_{\xi=1}^{m} \sum_{\zeta=1}^{m} d\left(\widetilde{\beta}_{\xi j}^{k}, \widetilde{\beta}_{\zeta j}^{k}\right)}{\sqrt{\sum_{j=1}^{n}\left(\sum_{\xi=1}^{m} \sum_{\zeta=1}^{m} d\left(\widetilde{\beta}_{\xi j}^{k}, \widetilde{\beta}_{\zeta j}^{k}\right)\right)^{2}}} .
$$

Finally, the optimal weight $w_{j}^{k}(j \in\{1,2, \ldots, n\}, k \in$ $\{1,2, \ldots, g\})$ is normalized as follows:

$$
\begin{aligned}
& \bar{w}_{j}^{k}=\frac{w_{j}^{k}}{\sum_{j=1}^{n} w_{j}^{k}}=\frac{\sum_{\xi=1}^{m} \sum_{\zeta=1}^{m} d\left(\widetilde{\beta}_{\xi j}^{k}, \widetilde{\beta}_{\zeta j}^{k}\right) / \sqrt{\sum_{j=1}^{n}\left(\sum_{\xi=1}^{m} \sum_{\zeta=1}^{m} d\left(\widetilde{\beta}_{\xi j}^{k} \widetilde{\beta}_{\zeta j}^{k}\right)\right)^{2}}}{\sum_{j=1}^{n} \sum_{\xi=1}^{m} \sum_{\zeta=1}^{m} d\left(\widetilde{\beta}_{\xi j}^{k}, \widetilde{\beta}_{\zeta j}^{k}\right) / \sqrt{\sum_{j=1}^{n}\left(\sum_{\xi=1}^{m} \sum_{\zeta=1}^{m} d\left(\widetilde{\beta}_{\xi j}^{k} \widetilde{\beta}_{\zeta j}^{k}\right)\right)^{2}}}=\frac{\sum_{\xi=1}^{m} \sum_{\zeta=1}^{m} d\left(\widetilde{\beta}_{\xi j}^{k}, \widetilde{\beta}_{\zeta j}^{k}\right)}{\sum_{j=1}^{n} \sum_{\xi=1}^{m} \sum_{\zeta=1}^{m} d\left(\widetilde{\beta}_{\xi j}^{k}, \widetilde{\beta}_{\zeta j}^{k}\right)}
\end{aligned}
$$

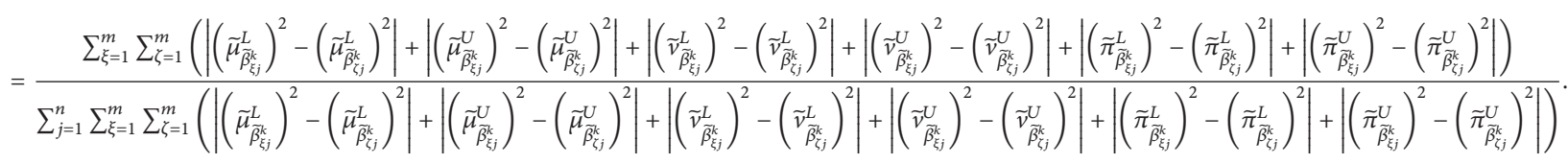

In addition, there are some real-life situations where the weights of criteria for each expert are not completely unknown but partially known. The structure forms of the partially known weights of criteria can 
be roughly divided into the following five basic forms [26-28]:

(1) a weak ranking: $\Gamma_{1}=\left\{w_{\xi}^{k} \geq w_{\zeta}^{k}\right\}$;

(2) a strict ranking: $\Gamma_{2}=\left\{w_{\xi}^{k}-w_{\zeta}^{k} \geq \varepsilon_{\xi \zeta}^{k}\right\}\left(\varepsilon_{\xi \zeta}^{k}>0\right)$;

(3) a ranking of differences: $\Gamma_{3}=\left\{w_{\xi}^{k}-w_{\zeta}^{k} \geq w_{f}^{k}-w_{g}^{k}\right\}(\xi \neq$ $\zeta \neq f \neq g)$;

(4) a ranking with multiples: $\Gamma_{4}=\left\{w_{\xi}^{k} \geq \varepsilon_{\xi \zeta}^{k} w_{\zeta}^{k}\right\}(0 \leq$ $\left.\varepsilon_{\xi \zeta}^{k} \leq 1\right)$

(5) An interval form: $\Gamma_{5}=\left\{\varepsilon_{\xi}^{k} \leq w_{\xi}^{k} \leq \kappa_{\xi}^{k}+\varepsilon_{\xi}^{k}\right\}\left(0 \leq \varepsilon_{\xi}^{k} \leq\right.$ $\left.\varepsilon_{\xi}^{k}+\kappa_{\xi}^{k} \leq 1\right)$.

The structure forms of the weights of criteria usually consist of several sets of the above basic sets or may contain all the five basic sets, which depend on the characteristic and need of the real-life decision problems. Let $\Gamma$ denote a set of the partially known weights of criteria and let $\Gamma=$ $\Gamma_{1} \cup \Gamma_{2} \cup \Gamma_{3} \cup \Gamma_{4} \cup \Gamma_{5}$. For these cases, we construct the following constrained optimization model to calculate the optimal weights of criteria for the expert $e_{k}$ :

$$
\begin{array}{ll}
\max & D^{k}=\sum_{j=1}^{n} \sum_{\xi=1}^{m} \sum_{\zeta=1}^{m} w_{j}^{k} d\left(\widetilde{\beta}_{\xi j}^{k}, \widetilde{\beta}_{\zeta j}^{k}\right) \\
\text { s.t } \quad & \mathbf{w}^{k} \in \Gamma, \\
& \sum_{j=1}^{n} w_{j}^{k}=1, \quad w_{j}^{k} \geq 0, \quad j \in\{1,2, \ldots, n\} .
\end{array}
$$

Model (MOD-2) can be easily executed by using MATLAB 7.4.0 or LINGO 11.0. By solving this model, we get the optimal solution $\mathbf{w}^{k}=\left(w_{1}^{k}, w_{2}^{k}, \ldots, w_{n}^{k}\right)^{T}$.

After obtaining the weights of criteria for each expert, we need to determine the weights of the criteria for the group. Denote the weight of the criterion $C_{j}(j \in\{1,2, \ldots, n\})$ for the group by $w_{j}^{*}$. Then, we further establish a minimizing consistency optimal model to calculate the optimal weights of criteria for the group as follows:

$$
\begin{array}{ll}
\min & Z\left(\mathbf{w}^{*}\right)=\sum_{j=1}^{n} \sum_{k=1}^{g} \lambda_{k}\left|w_{j}^{k}-w_{j}^{*}\right| \\
\text { s.t. } & \sum_{j=1}^{n} w_{j}^{*}=1, \quad w_{j}^{*} \geq 0, \quad j \in\{1,2, \ldots, n\} .
\end{array}
$$

To solve model (MOD-3), let

$$
\begin{aligned}
& \phi_{j}^{k}=\frac{1}{2}\left(\left|w_{j}^{k}-w_{j}^{*}\right|+\left(w_{j}^{k}-w_{j}^{*}\right)\right), \\
& \varphi_{j}^{k}=\frac{1}{2}\left(\left|w_{j}^{k}-w_{j}^{*}\right|-\left(w_{j}^{k}-w_{j}^{*}\right)\right) .
\end{aligned}
$$

Then, the optimal model (MOD-3) is transformed into the following line programming model:

$$
\begin{array}{ll}
\min \quad & Z\left(\mathbf{w}^{*}\right)=\sum_{j=1}^{n} \sum_{k=1}^{g} \lambda_{k}\left(\phi_{j}^{k}+\varphi_{j}^{k}\right) \\
\text { s.t. } \quad & w_{j}^{k}-w_{j}^{*}-\phi_{j}^{k}+\varphi_{j}^{k}=0 ; \\
& j \in\{1,2, \ldots, n\}, k \in\{1,2, \ldots, g\}, \\
& \phi_{j}^{k} \geq 0, \\
& \varphi_{j}^{k} \geq 0, \\
& \phi_{j}^{k} \varphi_{j}^{k}=0 ; \\
& j \in\{1,2, \ldots, n\}, k \in\{1,2, \ldots, g\}, \\
& \sum_{j=1}^{n} w_{j}^{*}=1, \quad w_{j}^{*} \geq 0 ; j \in\{1,2, \ldots, n\} .
\end{array}
$$

Model (MOD-4) can be easily executed by using MATLAB 7.4.0 or LINGO 11.0. By solving this optimal model, we get the optimal solution $\mathbf{w}^{*}=\left(w_{1}^{*}, w_{2}^{*}, \ldots, w_{n}^{*}\right)^{T}$.

3.3. The IVPF-WAA Operator for Determining the Ranking Order of Alternatives. After obtaining the optimal weights of criteria for the group, analogous to the literature $[22,23]$ we usually need to aggregate the given decision information so as to get the overall preference value of each alternative and the decision can be made. On the basis of the basic operational laws of IVPFNs introduced in Definition 6, in what follows we define an operator for aggregating IVPFNs.

Definition 15. Let $\widetilde{\beta}_{j}(j=1,2, \ldots, n)$ be a collection of IVPFNs, let $\mathbf{w}=\left(w_{1}, w_{2}, \ldots, w_{n}\right)^{T}$ be the weight vector of $\widetilde{\beta}_{j}(j=1,2, \ldots, n)$ with $w_{j} \in[0,1]$ and $\sum_{j=1}^{n} w_{j}=1$, and let IVPF-WAA : $\widetilde{\Theta}^{n} \rightarrow \widetilde{\Theta}$. If

$$
\begin{aligned}
& \operatorname{IVPF}-\mathrm{WAA}\left(\widetilde{\beta}_{1}, \widetilde{\beta}_{2}, \ldots, \widetilde{\beta}_{n}\right) \\
& \quad=w_{1} \widetilde{\beta}_{1} \oplus w_{2} \widetilde{\beta}_{2} \oplus \cdots \oplus w_{n} \widetilde{\beta}_{n}
\end{aligned}
$$

then the function IVPF-WAA is called an interval-valued Pythagorean fuzzy weighted average aggregating (IVPFWAA) operator.

Theorem 16. Let $\widetilde{\beta}_{j}=\widetilde{P}\left(\left[\widetilde{\mu}_{\widetilde{\beta}_{j}}^{L}, \widetilde{\mu}_{\widetilde{\beta}_{j}}^{U}\right],\left[\widetilde{v}_{\widetilde{\beta}_{j}}^{L}, \widetilde{v}_{\widetilde{\beta}_{j}}^{U}\right]\right)(j=1,2, \ldots, n)$ be a collection of IVPFNs; the aggregated value by using (21) is still an IVPFN; namely,

$$
\begin{aligned}
& I V P F-W A A\left(\tilde{\beta}_{1}, \tilde{\beta}_{2}, \ldots, \tilde{\beta}_{n}\right) \\
& \quad=\widetilde{P}\left(\left[\sqrt{1-\prod_{j=1}^{n}\left(1-\left(\tilde{\mu}_{\tilde{\beta}_{j}}^{L}\right)^{2}\right)^{w_{j}}},\right.\right.
\end{aligned}
$$




$$
\begin{aligned}
& \left.\sqrt{1-\prod_{j=1}^{n}\left(1-\left(\tilde{\mu}_{\tilde{\beta}_{j}}^{U}\right)^{2}\right)^{w_{j}}}\right],\left[\prod_{j=1}^{n}\left(\widetilde{v}_{\tilde{\beta}_{j}}^{L}\right)^{w_{j}},\right. \\
& \left.\left.\prod_{j=1}^{n}\left(\widetilde{v}_{\tilde{\beta}_{j}}^{U}\right)^{w_{j}}\right]\right)
\end{aligned}
$$

where $w_{j}$ indicates the importance degree of $\widetilde{\beta}_{j}$, satisfying $w_{j} \geq$ $0(j=1,2, \ldots, n)$ and $\sum_{j=1}^{n} w_{j}=1$.

Proof. By mathematical induction, the following results are obtained.

(1) If $n=2$, then

$$
\operatorname{IVPF}-\mathrm{WAA}\left(\widetilde{\beta}_{1}, \widetilde{\beta}_{2}\right)=w_{1} \widetilde{\beta}_{1} \oplus w_{2} \widetilde{\beta}_{2}
$$

According to operational law (3) in Definition 6, we have

$$
\begin{aligned}
& w_{1} \widetilde{\beta}_{1}=\widetilde{P}\left(\left[\sqrt{1-\left(1-\left(\widetilde{\mu}_{\tilde{\beta}_{1}}^{L}\right)^{2}\right)^{w_{1}}},\right.\right. \\
& \sqrt{\left.\left.1-\left(1-\left(\tilde{\mu}_{\tilde{\beta}_{1}}^{U}\right)^{2}\right)^{w_{1}}\right],\left[\left(\widetilde{v}_{\tilde{\beta}_{1}}^{L}\right)^{w_{1}},\left(\widetilde{v}_{\widetilde{\beta}_{1}}^{U}\right)^{w_{1}}\right]\right),} \\
& w_{2} \widetilde{\beta}_{2}=\widetilde{P}\left(\left[\sqrt{1-\left(1-\left(\tilde{\mu}_{\tilde{\beta}_{2}}^{L}\right)^{2}\right)^{w_{2}}},\right.\right. \\
& \sqrt{\left.\left.1-\left(1-\left(\widetilde{\mu}_{\tilde{\beta}_{2}}^{U}\right)^{2}\right)^{w_{2}}\right],\left[\left(\widetilde{v}_{\tilde{\beta}_{2}}^{L}\right)^{w_{2}},\left(\widetilde{v}_{\tilde{\beta}_{2}}^{U}\right)^{w_{2}}\right]\right),}
\end{aligned}
$$

and, by operational law (1) in Definition 6, we have

$$
\begin{aligned}
& w_{1} \widetilde{\beta}_{1} \oplus w_{2} \widetilde{\beta}_{2}=\widetilde{P}\left(\left[\sqrt{1-\left(1-\left(\widetilde{\mu}_{\tilde{\beta}_{1}}^{L}\right)^{2}\right)^{w_{1}}+1-\left(1-\left(\widetilde{\mu}_{\tilde{\beta}_{2}}^{L}\right)^{2}\right)^{w_{2}}-\left(1-\left(1-\left(\widetilde{\mu}_{\tilde{\beta}_{1}}^{L}\right)^{2}\right)^{w_{1}}\right)\left(1-\left(1-\left(\widetilde{\mu}_{\tilde{\beta}_{2}}^{L}\right)^{2}\right)^{w_{2}}\right)},\right.\right. \\
& \left.\sqrt{1-\left(1-\left(\widetilde{\mu}_{\tilde{\beta}_{1}}^{U}\right)^{2}\right)^{w_{1}}+1-\left(1-\left(\widetilde{\mu}_{\widetilde{\beta}_{2}}^{U}\right)^{2}\right)^{w_{2}}-\left(1-\left(1-\left(\widetilde{\mu}_{\tilde{\beta}_{1}}^{U}\right)^{2}\right)^{w_{1}}\right)\left(1-\left(1-\left(\widetilde{\mu}_{\tilde{\beta}_{2}}^{U}\right)^{2}\right)^{w_{2}}\right)}\right],\left[\left(\widetilde{v}_{\widetilde{\beta}_{1}}^{L}\right)^{w_{1}}\left(\widetilde{v}_{\widetilde{\beta}_{2}}^{L}\right)^{w_{2}},\right.
\end{aligned}
$$

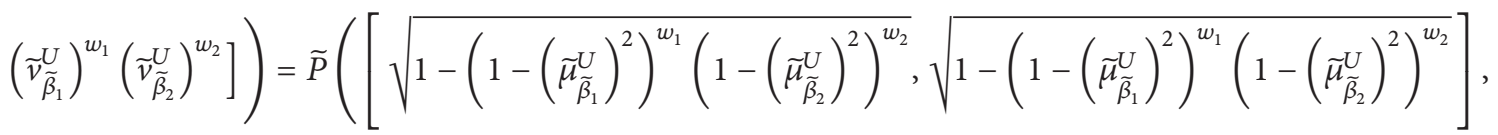

$$
\begin{aligned}
& \left.\left[\left(\widetilde{v}_{\widetilde{\beta}_{1}}^{L}\right)^{w_{1}}\left(\widetilde{v}_{\widetilde{\beta}_{2}}^{L}\right)^{w_{2}},\left(\widetilde{v}_{\widetilde{\beta}_{1}}^{U}\right)^{w_{1}}\left(\widetilde{v}_{\widetilde{\beta}_{2}}^{U}\right)^{w_{2}}\right]\right)
\end{aligned}
$$

Thus, (22) holds.

(2) We suppose (22) holds for $n=k$; that is,

$\operatorname{IVPF}-\mathrm{WAA}\left(\tilde{\beta}_{1}, \tilde{\beta}_{2}, \ldots, \widetilde{\beta}_{k}\right)$

$$
=\widetilde{P}\left(\left[\sqrt{1-\prod_{j=1}^{k}\left(1-\left(\tilde{\mu}_{\tilde{\beta}_{j}}^{L}\right)^{2}\right)^{w_{j}}}\right.\right.
$$

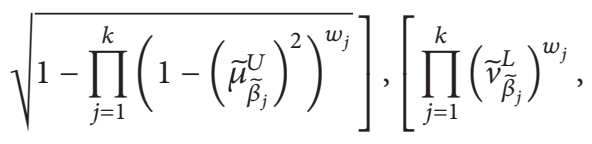$$
\left.\left.\prod_{j=1}^{k}\left(\widetilde{v}_{\widetilde{\beta}_{j}}\right)^{w_{j}}\right]\right)
$$

Then, if $n=k+1$, by operational laws (1) and (3) in Definition 6, we have

$w_{k+1} \widetilde{\beta}_{k+1}=\widetilde{P}\left(\left[\sqrt{1-\left(1-\left(\tilde{\mu}_{\tilde{\beta}_{k+1}}^{L}\right)^{2}\right)^{w_{k+1}}}, \sqrt{1-\left(1-\left(\tilde{\mu}_{\tilde{\beta}_{k+1}}^{U}\right)^{2}\right)^{w_{k+1}}}\right],\left[\left(\widetilde{v}_{\widetilde{\beta}_{k+1}}^{L}\right)^{w_{k+1}},\left(\widetilde{v}_{\tilde{\beta}_{k+1}}^{U}\right)^{w_{k+1}}\right]\right)$,

$\operatorname{IVPF}-W A A\left(\widetilde{\beta}_{1}, \widetilde{\beta}_{2}, \ldots, \widetilde{\beta}_{k+1}\right)=\left(w_{1} \widetilde{\beta}_{1} \oplus w_{2} \widetilde{\beta}_{2} \oplus \cdots \oplus w_{k} \widetilde{\beta}_{k}\right) \oplus w_{k+1} \widetilde{\beta}_{k+1}$ 


$$
\begin{aligned}
& =\widetilde{P}\left(\left[\sqrt{1-\prod_{j=1}^{k}\left(1-\left(\widetilde{\mu}_{\tilde{\beta}_{j}}^{L}\right)^{2}\right)^{w_{j}}+1-\left(1-\left(\widetilde{\mu}_{\tilde{\beta}_{k+1}}^{L}\right)^{2}\right)^{w_{k+1}}-\left(1-\prod_{j=1}^{k}\left(1-\left(\widetilde{\mu}_{\tilde{\beta}_{j}}^{L}\right)^{2}\right)^{w_{j}}\right)\left(1-\left(1-\left(\widetilde{\mu}_{\widetilde{\beta}_{k+1}}^{L}\right)^{2}\right)^{w_{k+1}}\right)},\right.\right. \\
& \sqrt{1-\prod_{j=1}^{k}\left(1-\left(\tilde{\mu}_{\tilde{\beta}_{j}}^{U}\right)^{2}\right)^{w_{j}}+1-\left(1-\left(\tilde{\mu}_{\tilde{\beta}_{k+1}}^{U}\right)^{2}\right)^{w_{k+1}}-\left(1-\prod_{j=1}^{k}\left(1-\left(\tilde{\mu}_{\tilde{\beta}_{j}}^{U}\right)^{2}\right)^{w_{j}}\right)\left(1-\left(1-\left(\tilde{\mu}_{\tilde{\beta}_{k+1}}^{U}\right)^{2}\right)^{w_{k+1}}\right)}, \\
& \left.\left[\left(\widetilde{v}_{\widetilde{\beta}_{k+1}}^{L}\right)^{w_{k+1}} \prod_{j=1}^{k}\left(\widetilde{v}_{\tilde{\beta}_{j}}^{L}\right)^{w_{j}},\left(\widetilde{v}_{\tilde{\beta}_{k+1}}^{U}\right)^{w_{k+1}} \prod_{j=1}^{k}\left(\widetilde{v}_{\tilde{\beta}_{j}}^{U}\right)^{w_{j}}\right]\right)=\widetilde{P}\left(\left[\sqrt{1-\prod_{j=1}^{k+1}\left(1-\left(\widetilde{\mu}_{\tilde{\beta}_{j}}^{L}\right)^{2}\right)^{w_{j}}}, \sqrt{1-\prod_{j=1}^{k+1}\left(1-\left(\tilde{\mu}_{\tilde{\beta}_{j}}^{U}\right)^{2}\right)^{w_{j}}}\right],\right. \\
& \left.\left[\prod_{j=1}^{k+1}\left(\widetilde{v}_{\widetilde{\beta}_{j}}^{L}\right)^{w_{j}}, \prod_{j=1}^{k+1}\left(\widetilde{v}_{\widetilde{\beta}_{j}}\right)^{w_{j}}\right]\right) .
\end{aligned}
$$

Therefore, (22) holds for $n=k+1$.

It is easy to conclude from (1) and (2) that (22) holds for any $n$.

This completes the proof of Theorem 16.

Example 17. For three IVPFNs, $\widetilde{\beta}_{1}=\widetilde{P}([0.8,0.9],[0.2,0.3])$, $\widetilde{\beta}_{2}=\widetilde{P}([0.6,0.7],[0.5,0.6])$, and $\widetilde{\beta}_{3}=\widetilde{P}([0.7,0.8],[0.4,0.5])$, and let $\mathbf{w}=(0.3,0.2,0.5)^{T}$. Then, according to (22), we can obtain

$\operatorname{IVPF}-\mathrm{WAA}\left(\widetilde{\beta}_{1}, \widetilde{\beta}_{2}, \widetilde{\beta}_{3}\right)$

$$
\begin{aligned}
& =\widetilde{P}\left(\left[\sqrt{1-\left(1-0.8^{2}\right)^{0.3}\left(1-0.6^{2}\right)^{0.2}\left(1-0.7^{2}\right)^{0.5}}\right.\right. \\
& \left.\sqrt{1-\left(1-0.9^{2}\right)^{0.3}\left(1-0.7^{2}\right)^{0.2}\left(1-0.8^{2}\right)^{0.5}}\right] \\
& \left.\left[0.2^{0.3} \times 0.5^{0.2} \times 0.4^{0.5}, 0.3^{0.3} \times 0.6^{0.2} \times 0.5^{0.5}\right]\right) \\
& =\widetilde{P}([0.7206,0.8254],[0.3397,0.4449])
\end{aligned}
$$

It is easy to show that the IVPF-WAA operator has some useful properties.

Proposition 18 (idempotency). Let $\widetilde{\beta}_{j}=\widetilde{P}\left(\left[\widetilde{\mu}_{\widetilde{\beta}_{j}}^{L}, \widetilde{\mu}_{\widetilde{\beta}_{j}}^{U}\right],\left[\widetilde{v}_{\widetilde{\beta}_{j}}^{L}\right.\right.$, $\left.\left.\widetilde{v}_{\widetilde{\beta}_{j}}^{U}\right]\right)(j=1,2, \ldots, n)$ be a collection of IVPFNs and let $\widetilde{\beta}$ be an IVPFN. If $\widetilde{\beta}_{j}=\widetilde{\beta}$, for all $j(j \in\{1,2, \ldots, n\})$, then

$$
\operatorname{IVPF}-W A A\left(\widetilde{\beta}_{1}, \widetilde{\beta}_{2}, \ldots, \widetilde{\beta}_{n}\right)=\widetilde{\beta} .
$$

Proposition 19 (bounded). Let $\widetilde{\beta}_{j}=\widetilde{P}\left(\left[\widetilde{\mu}_{\widetilde{\beta}_{j}}^{L}, \widetilde{\mu}_{\widetilde{\beta}_{j}}^{U}\right],\left[\widetilde{v}_{\widetilde{\beta}_{j}}^{L}\right.\right.$, $\left.\left.\widetilde{v}_{\widetilde{\beta}_{j}}^{U}\right]\right)(j=1,2, \ldots, n)$ be a collection of IVPFNs, and $\widetilde{\beta}^{-}=$ $\min _{j=1}^{n} \widetilde{\beta}_{j}$ and $\widetilde{\beta}^{+}=\max _{j=1}^{n} \tilde{\beta}_{j}$. Then,

$$
\widetilde{\beta}^{-} \leq \operatorname{IVPF}-W A A\left(\widetilde{\beta}_{1}, \widetilde{\beta}_{2}, \ldots, \widetilde{\beta}_{n}\right) \leq \widetilde{\beta}^{+} .
$$

Proposition 20 (monotonicity). Let $\widetilde{\beta}_{j}(j=1,2, \ldots, n)$ and $\tilde{\gamma}_{j}(j=1,2, \ldots, n)$ be two collections of IVPFNs. If $\widetilde{\beta}_{j} \leq \tilde{\gamma}_{j}$ for all $j(j \in\{1,2, \ldots, n\})$, then

$$
\begin{aligned}
& \operatorname{IVPF-WAA}\left(\widetilde{\beta}_{1}, \widetilde{\beta}_{2}, \ldots, \widetilde{\beta}_{n}\right) \\
& \quad \leq \operatorname{IVPF-WAA}\left(\tilde{\gamma}_{1}, \tilde{\gamma}_{2}, \ldots, \tilde{\gamma}_{n}\right) .
\end{aligned}
$$

Proposition 21 (IVPF-AA operator). If $\mathbf{w}=$ $(1 / n, 1 / n, \ldots, 1 / n)^{T}$, then IVPF-WAA operator is reduced to the interval-valued Pythagorean fuzzy average aggregating (IVPF-AA) operator; that is,

$$
\begin{aligned}
& I V P F-W A A\left(\widetilde{\beta}_{1}, \widetilde{\beta}_{2}, \ldots, \widetilde{\beta}_{n}\right)=\operatorname{IVPF}-W A\left(\widetilde{\beta}_{1}, \widetilde{\beta}_{2}, \ldots,\right. \\
& \left.\widetilde{\beta}_{n}\right)=\widetilde{P}\left(\left[\sqrt{1-\prod_{j=1}^{n}\left(1-\left(\tilde{\mu}_{\tilde{\beta}_{j}}^{L}\right)^{2}\right)^{1 / n}},\right.\right. \\
& \left.\sqrt{1-\prod_{j=1}^{n}\left(1-\left(\tilde{\mu}_{\widetilde{\beta}_{j}}^{U}\right)^{2}\right)^{1 / n}}\right],\left[\prod_{j=1}^{n}\left(\widetilde{v}_{\tilde{\beta}_{j}}^{L}\right)^{1 / n},\right. \\
& \left.\left.\prod_{j=1}^{n}\left(\widetilde{v}_{\tilde{\beta}_{j}}\right)^{1 / n}\right]\right) .
\end{aligned}
$$

In what follows, we employ the proposed IVPF-WAA operator as the main aggregation operator to obtain the comprehensive preference value of each alternative. By (22), all individual IVPF decision matrices $\mathscr{P}^{k}=\left(\widetilde{\beta}_{i j}^{k}\right)_{m \times n}(k \in$ $\{1,2, \ldots, g\})$ can be aggregated into the collective IVPF decision matrix $\mathscr{P}=\left(\widetilde{\mathscr{B}}_{i j}\right)_{m \times n}$, where

$$
\begin{aligned}
\widetilde{\mathscr{B}}_{i j} & =\lambda_{1} \widetilde{\beta}_{i j}^{1} \oplus \lambda_{2} \widetilde{\beta}_{i j}^{2} \oplus \cdots \oplus \lambda_{g} \widetilde{\beta}_{i j}^{g} \\
& =\widetilde{P}\left(\left[\sqrt{1-\prod_{k=1}^{g}\left(1-\left(\widetilde{u}_{\beta_{i j}^{k}}^{L}\right)^{2}\right)^{\lambda_{k}}},\right.\right.
\end{aligned}
$$




$$
\begin{aligned}
& \left.\sqrt{1-\prod_{k=1}^{g}\left(1-\left(\widetilde{u}_{\beta_{i j}^{k}}^{U}\right)^{2}\right)^{\lambda_{k}}}\right],\left[\prod_{k=1}^{g}\left(\widetilde{v}_{\beta_{i j}^{k}}^{L}\right)^{\lambda_{k}},\right. \\
& \left.\prod_{k=1}^{g}\left(\widetilde{v}_{\beta_{i j}^{k}}^{U}\right)^{\lambda_{k}}\right] .
\end{aligned}
$$

Then, the comprehensive preference value of the alternative $A_{i}(i \in\{1,2, \ldots, m\})$ can be calculated by using the following expression:

$$
\begin{aligned}
& \widetilde{\mathscr{D}}_{i}=w_{1} \widetilde{\mathscr{B}}_{i 1} \oplus w_{2} \widetilde{\mathscr{B}}_{i 2} \oplus \cdots \oplus w_{n} \widetilde{\mathscr{B}}_{i n} \\
&=\widetilde{P}\left(\left[\sqrt{1-\prod_{j=1}^{n}\left(1-\left(\widetilde{u}_{\widetilde{\mathscr{B}}_{i j}}^{L}\right)^{2}\right)^{w_{j}}},\right.\right. \\
&\left.\sqrt{1-\prod_{j=1}^{n}\left(1-\left(\widetilde{u}_{\widetilde{\mathscr{B}}_{i j}}^{U}\right)^{2}\right)^{w_{j}}}\right],\left[\prod_{j=1}^{n}\left(\widetilde{v}_{\widetilde{\mathscr{B}}_{i j}}^{L}\right)^{w_{j}},\right. \\
&\left.\left.\prod_{j=1}^{n}\left(\widetilde{v}_{\mathscr{\mathscr { B }}_{i j}}^{U}\right)^{w_{j}}\right]\right) .
\end{aligned}
$$

By Definition 9, we can compare the magnitude of the comprehensive preference value of each alternative and further determine the best alternative.

The algorithm of the proposed approach can be summarized as follows.

Step 1. Construct the interval-valued Pythagorean fuzzy decision matrix $\mathscr{P}^{k}=\widetilde{P}\left(\left[\widetilde{u}_{\widetilde{\beta}_{i j}^{k}}^{L}, \tilde{u}_{\widetilde{\beta}_{i j}^{k}}^{U}\right],\left[\widetilde{v}_{\widetilde{\beta}_{i j}^{k}}^{L}, \widetilde{v}_{\widetilde{\beta}_{i j}^{k}}^{U}\right]\right)_{m \times n}(k \in$ $\{1,2, \ldots, g\})$.

Step 2. Determine the weights of criteria for each expert by solving (19) or model (MOD-2).

Step 3. Calculate the weights of criteria for the group by solving model (MOD-4).

Step 4. Compute the comprehensive preference values of the alternative $A_{i}(i=1,2, \ldots, m)$ by using (33) and (34).

Step 5. Determine the optimal ranking order of the alternatives by comparing the magnitude of the comprehensive preference values of all alternatives based on Definition 9 and further identify the optimal alternative.

\section{Illustrative Example}

In this section, an illustrating case concerning the risk evaluation of technological innovation in high-tech enterprises is provided to demonstrate the proposed method.

4.1. Description. Technological innovation is the foundation of high-tech enterprises' long term stable development. The risk evaluation of technologic innovation plays an important role in high-tech enterprises' development. Usually, this risk evaluation involves multiple experts and multiple conflicting evaluation indices (criteria), which can be regarded as an MCGDM problem. In this section, we employ the proposed method to help the decision maker to select the optimal hightech enterprise with the lowest risk of technologic innovation from four potential high-tech enterprises $\left\{A_{1}, A_{2}, A_{3}, A_{4}\right\}$. After analyzing these high-tech enterprises, the criteria considered for the risk assessment of technologic innovation are as follows: policy risk $\left(C_{1}\right)$, financial risk $\left(C_{2}\right)$, technological risk $\left(C_{3}\right)$, production risk $\left(C_{4}\right)$, market risk $\left(C_{5}\right)$, and managerial risk $\left(C_{6}\right)$. Three experts $\left\{e_{1}, e_{2}, e_{3}\right\}$ who are specializing in risk evaluation of technologic innovation are invited to evaluate these four high-tech enterprises according to these six evaluation criteria. We assume that the weight vector of experts is given in advance as $\boldsymbol{\lambda}=(0.4,0.35,0.25)^{T}$, and the weights of criteria are completely unknown. The assessment values of alternatives with respect to criteria provided by the experts are assumed to be represented by IVPFNs as shown in interval-valued Pythagorean fuzzy group decision matrix given in Table 1.

The left-top cell $\widetilde{P}([0.8,0.9],[0.2,0.3])$ in Table 1 can be explained, where the alternative $A_{1}$ is an excellent alternative for the expert $e_{1}$ on the criterion $C_{1}$ with a margin of 80$90 \%$ and simultaneously $A_{1}$ is not an excellent choice with a chance between $20 \%$ and $30 \%$, and the others in Table 1 have similar meanings.

4.2. Decision Process. In the following, we use the proposed method to solve the problem mentioned in Section 4.1. Firstly, we use (19) to calculate the weights of criteria for each expert as follows:

$$
\begin{aligned}
& \mathbf{w}^{1} \\
& \quad=(0.2064,0.1505,0.1325,0.1145,0.1897,0.2064)^{T}, \\
& \mathbf{w}^{2} \\
& \quad=(0.1433,0.1841,0.1361,0.2320,0.1613,0.1433)^{T}, \\
& \mathbf{w}^{3} \\
& =(0.1681,0.2232,0.1668,0.1257,0.1482,0.1681)^{T} .
\end{aligned}
$$

Secondly, in the sense of model (MOD-4), we construct the following linear programming model to derive the weights of criteria for the group:

$$
\begin{aligned}
\min & Z\left(\mathbf{w}^{*}\right) \\
& =0.4 \times\left(\phi_{1}^{1}+\varphi_{1}^{1}+\phi_{2}^{1}+\varphi_{2}^{1}+\phi_{3}^{1}+\varphi_{3}^{1}+\phi_{4}^{1}+\varphi_{4}^{1}+\phi_{5}^{1}+\varphi_{5}^{1}+\phi_{6}^{1}+\varphi_{6}^{1}\right)+0.35
\end{aligned}
$$




$$
\begin{aligned}
& \times\left(\phi_{1}^{2}+\varphi_{1}^{2}+\phi_{2}^{2}+\varphi_{2}^{2}+\phi_{3}^{2}+\varphi_{3}^{2}+\phi_{4}^{2}+\varphi_{4}^{2}+\phi_{5}^{2}+\varphi_{5}^{2}+\phi_{6}^{2}+\varphi_{6}^{2}\right)+0.25 \\
& \times\left(\phi_{1}^{3}+\varphi_{1}^{3}+\phi_{2}^{3}+\varphi_{2}^{3}+\phi_{3}^{3}+\varphi_{3}^{3}+\phi_{4}^{3}+\varphi_{4}^{3}+\phi_{5}^{3}+\varphi_{5}^{3}+\phi_{6}^{3}+\varphi_{6}^{3}\right) \\
& \text { s.t. } \quad 0.2064-w_{1}^{*}-\phi_{1}^{1}+\varphi_{1}^{1}=0 \text {; } \\
& 0.1505-w_{2}^{*}-\phi_{2}^{1}+\varphi_{2}^{1}=0 \\
& 0.1325-w_{3}^{*}-\phi_{3}^{1}+\varphi_{3}^{1}=0 \\
& 0.1145-w_{4}^{*}-\phi_{4}^{1}+\varphi_{4}^{1}=0 ; \\
& 0.1897-w_{5}^{*}-\phi_{5}^{1}+\varphi_{5}^{1}=0 \text {; } \\
& 0.2064-w_{6}^{*}-\phi_{6}^{1}+\varphi_{6}^{1}=0 \\
& 0.1433-w_{1}^{*}-\phi_{1}^{2}+\varphi_{1}^{2}=0 ; \\
& 0.1841-w_{2}^{*}-\phi_{2}^{2}+\varphi_{2}^{2}=0 ; \\
& 0.1361-w_{3}^{*}-\phi_{3}^{2}+\varphi_{3}^{2}=0 ; \\
& 0.2320-w_{4}^{*}-\phi_{4}^{2}+\varphi_{4}^{2}=0 \text {; } \\
& 0.1613-w_{5}^{*}-\phi_{5}^{2}+\varphi_{5}^{2}=0 ; \\
& 0.1433-w_{6}^{*}-\phi_{6}^{2}+\varphi_{6}^{2}=0 ; \\
& 0.1681-w_{1}^{*}-\phi_{1}^{3}+\varphi_{1}^{3}=0 ; \\
& 0.2232-w_{2}^{*}-\phi_{2}^{3}+\varphi_{2}^{3}=0 ; \\
& 0.1668-w_{3}^{*}-\phi_{3}^{3}+\varphi_{3}^{3}=0 ; \\
& 0.1257-w_{4}^{*}-\phi_{4}^{3}+\varphi_{4}^{3}=0 ; \\
& 0.1482-w_{5}^{*}-\phi_{5}^{3}+\varphi_{5}^{3}=0 ; \\
& 0.1681-w_{6}^{*}-\phi_{6}^{3}+\varphi_{6}^{3}=0 ; \\
& \phi_{j}^{k} \geq 0 \text {, } \\
& \varphi_{j}^{k} \geq 0, \\
& \phi_{j}^{k} \varphi_{j}^{k}=0 ; \\
& j \in\{1,2, \ldots, 6\}, k \in\{1,2,3\} \\
& w_{1}^{*}+w_{2}^{*}+w_{3}^{*}+w_{4}^{*}+w_{5}^{*}+w_{6}^{*}=1 \\
& w_{j}^{*}>0, \quad j \in\{1,2, \ldots, 6\} \text {. }
\end{aligned}
$$

(MOD-5)

By solving model (MOD-5) using LINGO 11.0, the following results are obtained:

$$
\begin{aligned}
& \mathbf{w}^{*} \\
& \quad=(0.1894,0.1841,0.1361,0.1257,0.1753,0.1894)^{T} .
\end{aligned}
$$

Meanwhile, we utilize (33) to aggregate all individual decision matrices into the collective decision matrix, and the results are listed in Table 2.

Finally, we employ (34) to aggregate the collective decision data in Table 2 to obtain the comprehensive preference values of alternatives as shown in Table 3. The scores of 


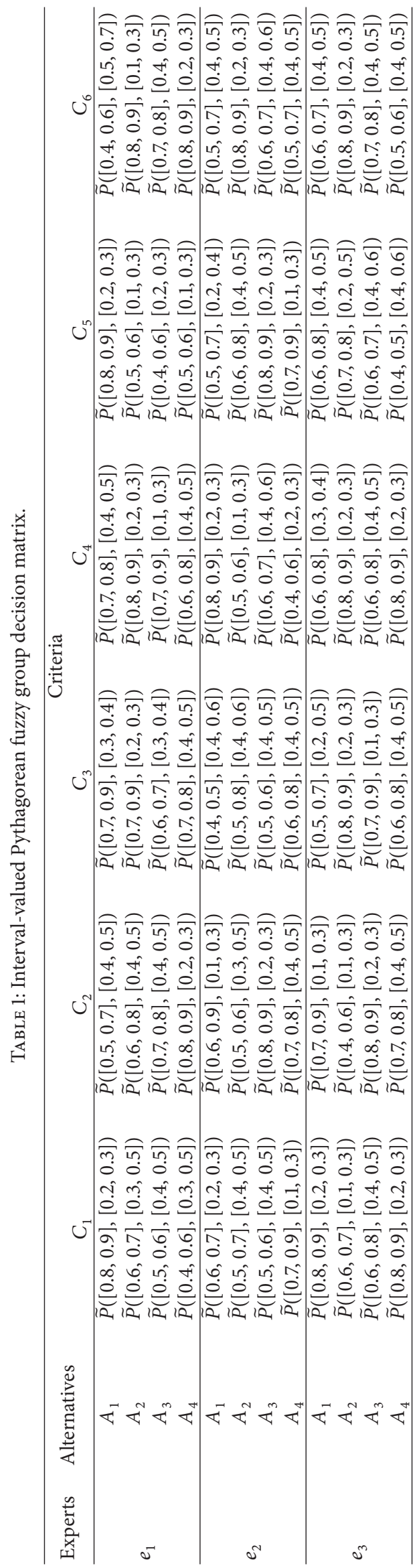




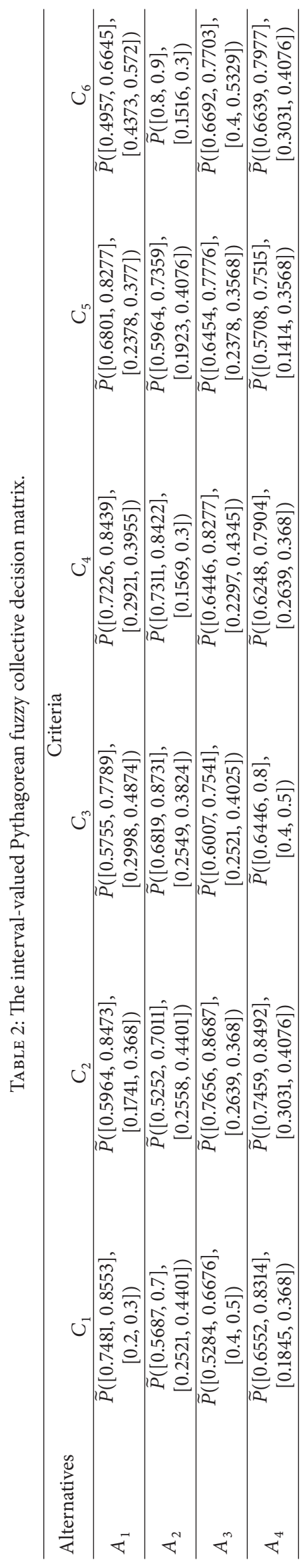


TABLE 3: The collective preference values, scores, and ranking of alternatives.

\begin{tabular}{lccc}
\hline Alternatives & Collective preference values & Scores & Ranking \\
\hline$A_{1}$ & $\widetilde{P}([0.6487,0.8123],[0.3031,0.4076])$ & 0.4249 & 3 \\
$A_{2}$ & $\widetilde{P}([0.6656,0.8076],[0.2067,0.3775])$ & 0.4550 & 1 \\
$A_{3}$ & $\widetilde{P}([0.6547,0.7863],[0.2963,0.4301])$ & 0.3872 & 4 \\
$A_{4}$ & $\widetilde{P}([0.6593,0.8078],[0.2464,0.3964])$ & 0.4347 & 2 \\
\hline
\end{tabular}

the comprehensive preference values of all alternatives are computed by (7). According to these score values, we can obtain the ranking of all alternatives as shown in Table 3 .

It can be easily seen from Table 3 that the optimal ranking order is $A_{2}>A_{4}>A_{1}>A_{3}$, and thus the best alternative is $A_{2}$. Apparently, the proposed method can deal effectively with the risk evaluation problem of technological innovation in high-tech enterprises and help the decision maker to select the optimal enterprise with the lowest risk of technological innovation. Additionally, the proposed method can also be applied in other MCGDM problems with incomplete weight information under interval-valued Pythagorean fuzzy environments.

4.3. Comparative Analysis. In this section, we chose the Pythagorean fuzzy TOPSIS approach proposed by Zhang and $\mathrm{Xu}$ [11] to conduct a comparative analysis in order to demonstrate the advantage of the proposed method. It is noted that the Pythagorean fuzzy TOPSIS approach is just suitable to deal with the MCDM problems with PFNs, but it fails to handle the above MCGDM problem with IVPFNs. Therefore, we extend Pythagorean fuzzy TOPSIS approach to tackle appropriately the MCGDM problems with IVPFNs.

In the extended Pythagorean fuzzy TOPSIS (we call it IVPF-TOPSIS) method, we first use the IVPF-WAA operator (i.e., (33)) to aggregate all individual IVPF decision matrices $\mathscr{P}^{k}=\left(\widetilde{\beta}_{i j}^{k}\right)_{m \times n}(k \in\{1,2, \ldots, g\})$ into the collective IVPF decision matrix $\mathscr{P}=\left(\widetilde{\mathscr{B}}_{i j}\right)_{m \times n}$. The aggregating results in the above decision problem are listed in Table 2 . Then, we assume that the IVPF positive ideal solution (IVPF-PIS) $A^{+}$and the IVPF negative ideal solution (IVPF-NIS) $A^{-}$are obtained as follows:

$$
\begin{aligned}
A^{+} & =\left\{\widetilde{\mathscr{B}}_{1}^{+}, \widetilde{\mathscr{B}}_{2}^{+}, \ldots, \widetilde{\mathscr{B}}_{n}^{+}\right\} \\
& =\{([1,1],[0,0]), \ldots,([1,1],[0,0])\}, \\
A^{-} & =\left\{\widetilde{\mathscr{B}}_{1}^{-}, \widetilde{\mathscr{B}}_{2}^{-}, \ldots, \widetilde{\mathscr{B}}_{n}^{-}\right\} \\
& =\{([0,0],[1,1]), \ldots,([0,0],[1,1])\} .
\end{aligned}
$$

Owing to the fact that the IVPF-TOPSIS method requires the experts to provide the weights of criteria in advance, we here assume that the weights of criteria for the group are given as

$$
\begin{aligned}
& \mathbf{w}^{*} \\
& \quad=(0.1894,0.1841,0.1361,0.1257,0.1753,0.1894)^{T} .
\end{aligned}
$$

TABLE 4: Closeness coefficients of alternatives along with the final ranking.

\begin{tabular}{lcccc}
\hline Alternatives & $S_{i}^{+}$ & $S_{i}^{-}$ & $\mathrm{CC}_{i}$ & Ranking \\
\hline$A_{1}$ & 0.4718 & 0.8684 & 0.648 & 3 \\
$A_{2}$ & 0.4742 & 0.9017 & 0.6554 & 2 \\
$A_{3}$ & 0.4874 & 0.8536 & 0.6365 & 4 \\
$A_{4}$ & 0.4606 & 0.8821 & 0.6569 & 1 \\
\hline
\end{tabular}

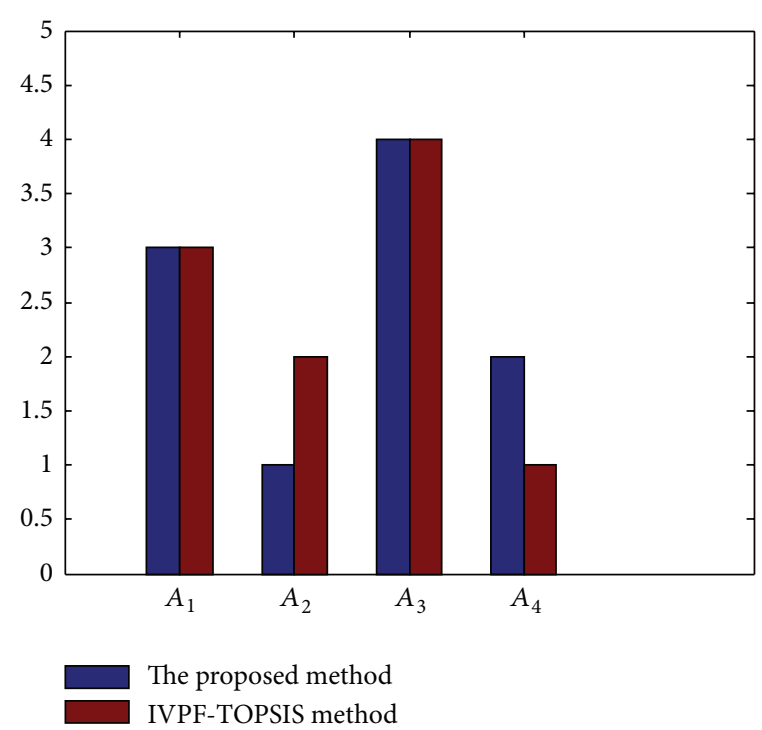

FIGURE 1: The pictorial representation of the rankings of alternatives.

Furthermore, using the interval-valued Pythagorean fuzzy distance measure (i.e., (10)) the separation measure $S_{i}^{+}$ between the alternative $A_{i}(i \in\{1,2, \ldots, m\})$ and IVPF-PIS $A^{+}$and the separation measures $S_{i}^{-}$between the alternative $A_{i}(i \in\{1,2, \ldots, m\})$ and the IVPF-NIS $A^{-}$are calculated, respectively. Then, the relative closeness coefficient of the alternative $A_{i}(i \in\{1,2, \ldots, m\})$ is defined as the following formula:

$$
\mathrm{CC}_{i}=\frac{S_{i}^{-}}{S_{i}^{+}+S_{i}^{-}} .
$$

Using (10) and (37)-(39), the corresponding separation measures $S_{i}^{+}$and $S_{i}^{-}$and the relative closeness coefficient $\mathrm{CC}_{i}$ in the above problem can be obtained, respectively. These results are presented in Table 4, together with the corresponding rankings of the alternatives on the basis of $\mathrm{CC}_{i}$.

From Table 4, it is easy to see that the optimal order for these four companies is $A_{4}>A_{2}>A_{1}>A_{3}$. To provide a better view of the comparison results, we put the results of the ranking of alternatives obtained by the proposed method and IVPF-TOPSIS approach into Figure 1.

From Figure 1, it is easy to see that the decision results of the proposed method are very little different from the results by using the IVPF-TOPSIS method. The difference is just the ranking order between $A_{2}$ and $A_{4}$; that is, $A_{2}>$ $A_{4}$, for the proposed method, and $A_{2} \prec A_{4}$ for the IVPFTOPSIS approach. The main reason is that the decision result 
of our proposed method is obtained by using the IVPF-WAA operator, while the result of the IVPF-TOPSIS approach is obtained on the basis of the distances between alternatives and the ideal solutions. Compared with the IVPF-TOPSIS approach, the proposed method does not require experts to provide the weights of criteria in advance, but it constructs two optimal models to determine objectively the weights, which avoids the subjective randomness of selecting the weights of criteria.

In addition, according to Table 4, it is noted that the relative closeness coefficients of alternatives $\left(\mathrm{CC}_{3}=0.6365<\right.$ $\left.\mathrm{CC}_{1}=0.6480<\mathrm{CC}_{2}=0.6554<\mathrm{CC}_{4}=0.6569\right)$ are low differences. It is not easy to conclude that the alternative $A_{4}$ is superior to $A_{2}$ based on their closeness coefficients $\left(\mathrm{CC}_{2}=\right.$ $\left.0.6554<\mathrm{CC}_{4}=0.6569\right)$ and the best alternative is $A_{4}$. The natural imprecision may be bigger than those differences. In other words, the ranking orders of alternatives based on their relative closeness coefficients which are low differences may not be quite reasonable. While, in our proposed method, the obtained ranking orders of alternatives are based on the scores of the collective preference values of alternatives. According to Table 3 , it is noted that the scores of the collective preference values of alternatives $\left(s\left(A_{3}\right)=0.3872<\right.$ $\left.s\left(A_{1}\right)=0.4249<s\left(A_{4}\right)=0.4347<s\left(A_{2}\right)=0.4550\right)$ are quite different. It is easily observed that the ranking order of alternatives is $A_{2}>A_{4}>A_{1}>A_{3}$ and the best alternative is $A_{2}$. Apparently, compared with the Pythagorean fuzzy TOPSIS approach [11], the decision results obtained by the proposed method are more effective and reasonable.

\section{Conclusions}

In this paper, we have developed a maximizing deviation method based on the IVPF-WAA operator to solve MCGDM problems with interval-valued Pythagorean fuzzy information. We first have defined the score and accuracy functions for IVPFNs and meanwhile presented a score and accuracy functions-based ranking method for comparing the magnitude of IVPFNs. Then, we have developed an IVPF-WAA operator to aggregate the given decision data in order to get the overall preference values of alternatives. We have also investigated some useful properties of IVPF-WAA operator. Afterwards, we have established an optimization model on the basis of the maximizing deviation method for determining the weights of criteria for each expert. Moreover, based on the derived weights of criteria for each expert, we have constructed a minimizing consistency optimal model to derive the weights of criteria for the group. At length, the proposed method is applied to solve the risk evaluation case of technological innovation in high-tech enterprises. Obviously, the proposed method can deal effectively with the interval-valued Pythagorean fuzzy MCGDM problems with incomplete weight information. Compared with the Pythagorean fuzzy TOPSIS approach [11], the main advantage of the proposed method is that it does not require experts to provide the weights of criteria in advance, but it constructs two optimal models to determine objectively the weights, which avoids the subjective randomness of selecting the weights of criteria, and meanwhile it can sufficiently consider the uncertainty and ambiguity inherent in the human decision process by utilizing the IVPFNs.

However, this study contains some limitations. One of the limitations of this study is that the experimental studies with different sizes are lacking. In future studies, we will focus on some additional experimental studies with different sizes of randomly generated problems and discuss how the weights of criteria and the ranking orders of alternatives are obtained. Another limitation is that the sensitivity analysis for the decision result is lacking in this paper. In the future studies, we will conduct the sensitivity analysis for the proposed method by modifying (i.e., increasing or decreasing) the interval-valued Pythagorean fuzzy decision data. In addition, in order to facilitate experts to make a reasonable decision, in the future studies, we will develop the corresponding decision support systems based on the proposed method to solve the real-world decision problems.

\section{Conflict of Interests}

The authors declare that there is no conflict of interests regarding the publication of this paper.

\section{Acknowledgments}

The authors are very grateful to the anonymous reviewers and the editor for their insightful and constructive comments and suggestions that have led to an improved version of this paper. The work was supported by the National Natural Science Foundation of China (nos. 71263020, 71363020 and 61363075), the National Social Science Foundation of China (no. 10CGL045), the Natural Science Foundation of Jiangxi Province of China (no. 20142BAB201009), and the Technology Landing Plan Project of Jiangxi Province of China (no. KJLD12064).

\section{References}

[1] L. A. Zadeh, "Fuzzy sets," Information and Control, vol. 8, pp. 338-353, 1965.

[2] R. E. Bellman and L. A. Zadeh, "Decision-making in a fuzzy environment," Management Science, vol. 17, pp. B141-B164, 1970.

[3] P. Liu, "Some hamacher aggregation operators based on the interval-valued intuitionistic fuzzy numbers and their application to group decision making," IEEE Transactions on Fuzzy Systems, vol. 22, no. 1, pp. 83-97, 2014.

[4] K. T. Atanassov, "Intuitionistic fuzzy sets," Fuzzy Sets and Systems, vol. 20, no. 1, pp. 87-96, 1986.

[5] G. Deschrijver and E. E. Kerre, "On the position of intuitionistic fuzzy set theory in the framework of theories modelling imprecision," Information Sciences, vol. 177, no. 8, pp. 1860-1866, 2007.

[6] F. Wang, S. Zeng, and C. Zhang, "A method based on intuitionistic fuzzy dependent aggregation operators for supplier selection," Mathematical Problems in Engineering, vol. 2013, Article ID 481202, 9 pages, 2013.

[7] J.-Q. Wang and H.-Y. Zhang, "Multicriteria decision-making approach based on atanassov's intuitionistic fuzzy sets with 
incomplete certain information on weights," IEEE Transactions on Fuzzy Systems, vol. 21, no. 3, pp. 510-515, 2013.

[8] Y. Li, Y. Shan, and P. Liu, "An extended TODIM method for group decision making with the interval intuitionistic fuzzy sets," Mathematical Problems in Engineering, vol. 2015, Article ID 672140, 9 pages, 2015.

[9] K. T. Atanassov, Intuitionistic Fuzzy Sets, vol. 35 of Studies in Fuzziness and Soft Computing, Springer, Heidelberg, Germany, 1999.

[10] R. R. Yager, "Pythagorean membership grades in multicriteria decision making," IEEE Transactions on Fuzzy Systems, vol. 22, no. 4, pp. 958-965, 2014.

[11] X. L. Zhang and Z. S. Xu, "Extension of TOPSIS to multiple criteria decision making with pythagorean fuzzy sets," International Journal of Intelligent Systems, vol. 29, no. 12, pp.1061-1078, 2014.

[12] R. R. Yager and A. M. Abbasov, "Pythagorean membership grades, complex numbers, and decision making," International Journal of Intelligent Systems, vol. 28, no. 5, pp. 436-452, 2013.

[13] G. Beliakov and S. James, "Averaging aggregation functions for preferences expressed as Pythagorean membership grades and fuzzy orthopairs," in Proceedings of the IEEE International Conference on Fuzzy Systems (FUZZ-IEEE '14), pp. 298-305, IEEE, July 2014.

[14] M. Z. Reformat and R. R. Yager, "Suggesting recommendations using pythagorean fuzzy sets illustrated using netflix movie data," in Information Processing and Management of Uncertainty in Knowledge-Based Systems, pp. 546-556, Springer, 2014.

[15] K. Atanassov, P. Vassilev, and R. Tcvetkov, Intuitionistic Fuzzy Sets, Measures and Integrals, "Prof. M. Drinov" Academic Publishing House, Sofia, Bulgaria, 2013.

[16] W. Pedrycz, Granular Computing: Analysis and Design of Intelligent Systems, CRC Press, Francis Taylor, Boca Raton, Fla, USA, 2013.

[17] T.-Y. Chen, "Interval-valued intuitionistic fuzzy QUALIFLEX method with a likelihood-based comparison approach for multiple criteria decision analysis," Information Sciences, vol. 261, pp. 149-169, 2014.

[18] X. Zhang, "Multicriteria Pythagorean fuzzy decision analysis: a hierarchical QUALIFLEX approach with the closeness indexbased ranking methods," Information Sciences, 2015.

[19] K. T. Atanassov and G. Gargov, "Interval valued intuitionistic fuzzy sets," Fuzzy Sets and Systems, vol. 31, no. 3, pp. 343-349, 1989.

[20] J. Wang, P. Wang, J. Wang, H. Zhang, and X. Chen, "Atanassov's interval-valued intuitionistic linguistic multicriteria group decision-making method based on the trapezium cloud model," IEEE Transactions on Fuzzy Systems, vol. 23, no. 3, pp. 542-554, 2015.

[21] K. T. Atanassov, On Intuitionistic Fuzzy Sets Theory, Springer, Berlin, Germany, 2012.

[22] Y. M. Wang, "Using the method of maximizing deviation to make decision for multiindices," Journal of Systems Engineering and Electronics in China, vol. 8, pp. 21-26, 1997.

[23] Z. B. Wu and Y. H. Chen, "The maximizing deviation method for group multiple attribute decision making under linguistic environment," Fuzzy Sets and Systems, vol. 158, no. 14, pp. 16081617, 2007.

[24] Z. Xu and X. Zhang, "Hesitant fuzzy multi-attribute decision making based on TOPSIS with incomplete weight information," Knowledge-Based Systems, vol. 52, pp. 53-64, 2013.
[25] G.-W. Wei, "Maximizing deviation method for multiple attribute decision making in intuitionistic fuzzy setting," Knowledge-Based Systems, vol. 21, no. 8, pp. 833-836, 2008.

[26] S. H. Kim and B. S. Ahn, "Interactive group decision making procedure under incomplete information," European Journal of Operational Research, vol. 116, no. 3, pp. 498-507, 1999.

[27] X. L. Zhang, Z. S. Xu, and H. Wang, "Heterogeneous multiple criteria group decision making with incomplete weight information: a deviation modeling approach," Information Fusion, vol. 25, pp. 49-62, 2015.

[28] X. L. Zhang and Z. S. Xu, "Soft computing based on maximizing consensus and fuzzy TOPSIS approach to interval-valued intuitionistic fuzzy group decision making," Applied Soft Computing Journal, vol. 26, pp. 42-56, 2015. 


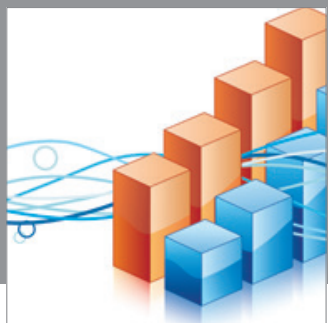

Advances in

Operations Research

mansans

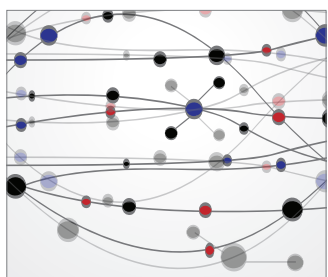

The Scientific World Journal
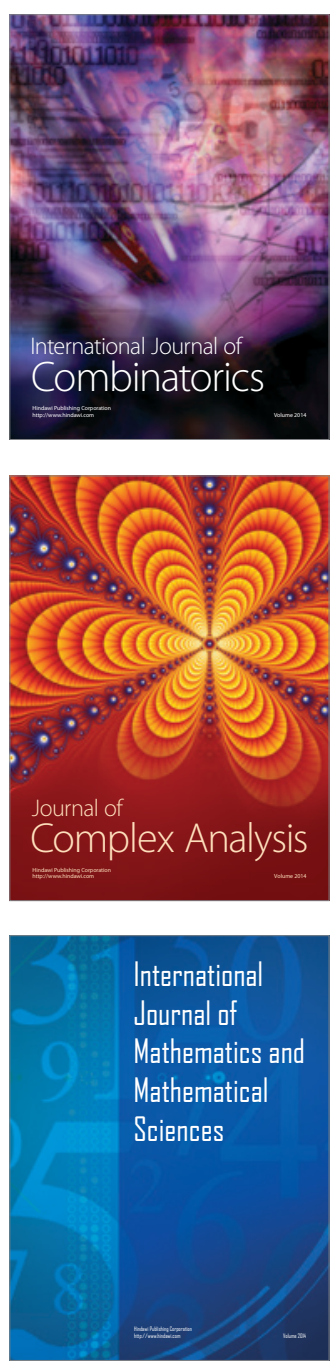
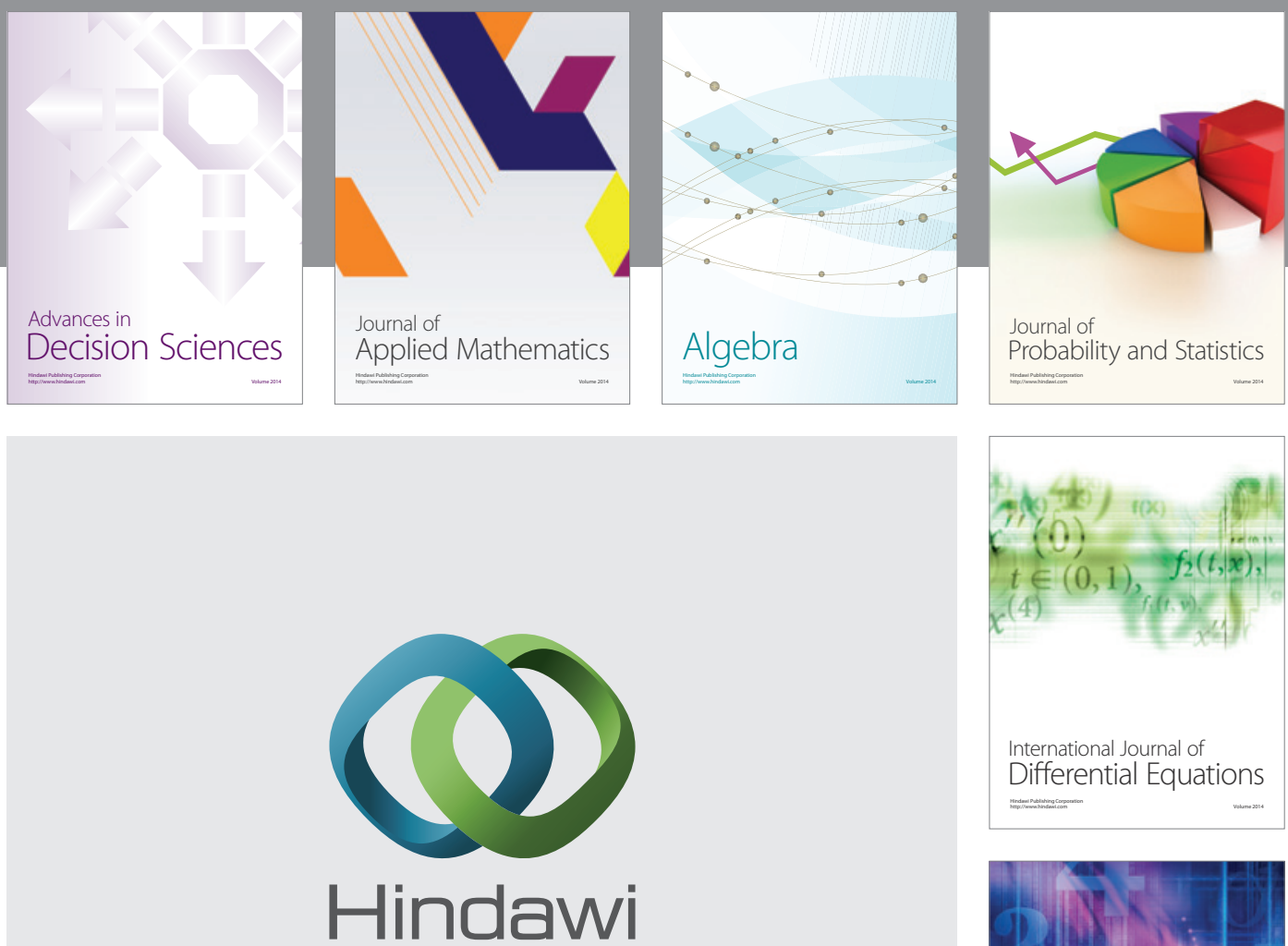

Submit your manuscripts at http://www.hindawi.com
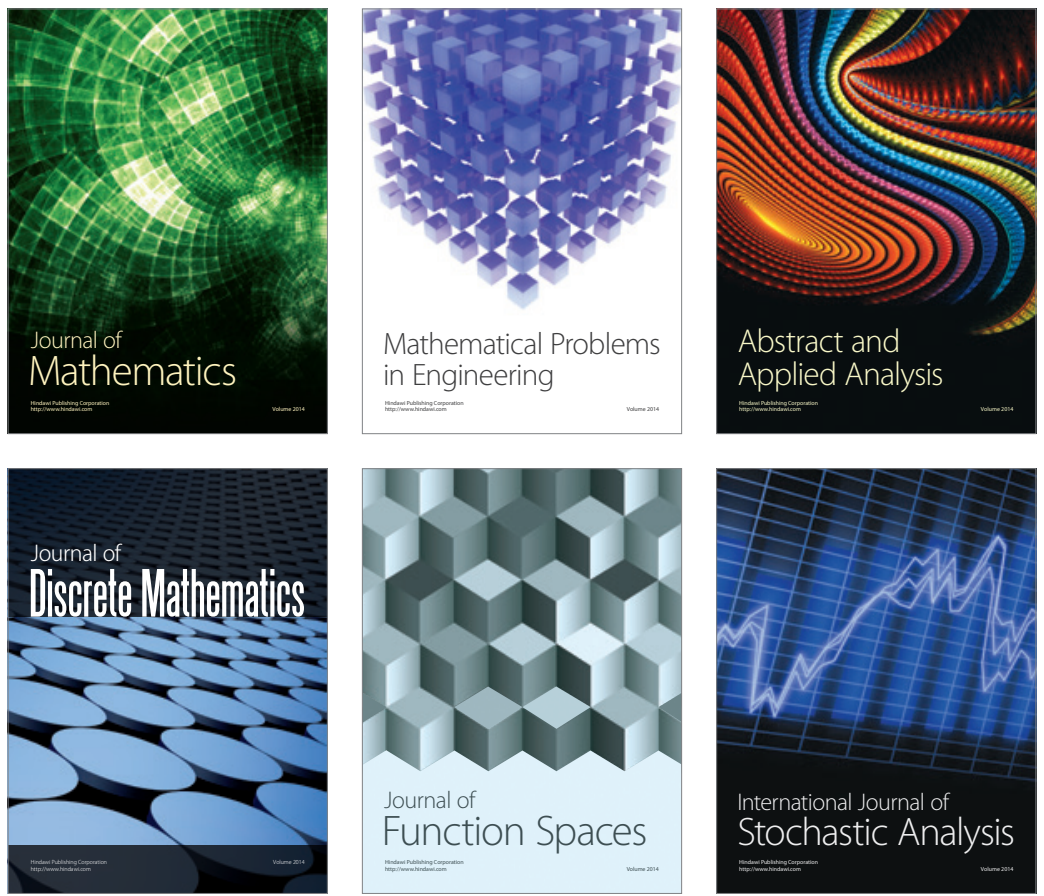

Journal of

Function Spaces

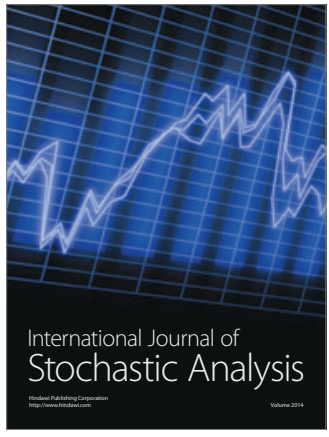

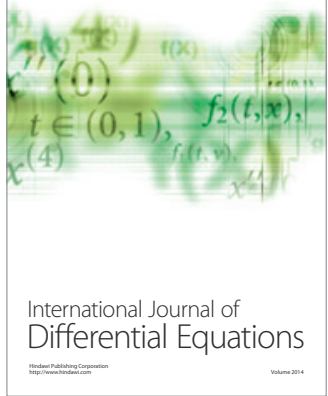
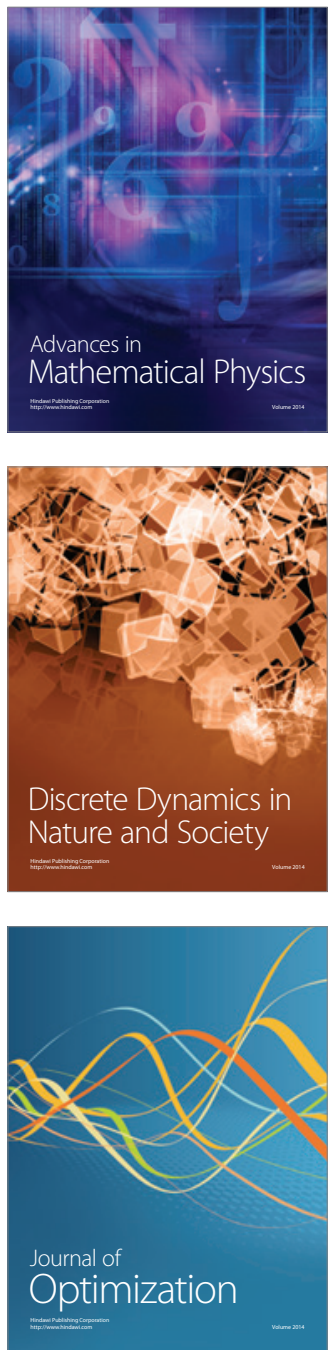